\title{
Abstracts of 16th Annual meeting of Paediatric Endoscopic Surgeons of India, 19-21 March 2021
}

E-PESICON 2021 - Abstracts 


\section{Paediatric Endoscopic Surgeons of India (PESI) Annual Conference-PESICON 2021}

The 16th Annual Conference of Paediatric Endoscopic Surgeons of India, PESICON 2021 was organized as a "first hybrid event" of Paediatric Endoscopic Surgeons of India-Indian Association of Paediatric Surgeons (PESI-IAPS) at Mumbai in collaboration with SRCC Children's Hospital Mumbai from 19th to 21st March 2021.

The event was a grand success with more than 200 national and international delegates attending the event on virtual platform. Day 1 had PESI-IAPS oration, Karl Storz best video award session and free paper sessions. Prof Anette Jacobsen from Singapore gave an oration titled "Paediatric Laparoscopy Surgery in Singapore- History and Developments".

Day 2 was "live operative workshop" in which total 14 minimal access surgeries were operated at 3 different centres (SRCC Children Hospital Mumbai, IGICH Bengaluru and AIIMS Jodhpur) and relayed live on virtual platform simultaneously. The day concluded with an excellent lecture by Dr. Dariusz Patkowski from Poland on his experience of thoracoscopic esophageal atresia repair.

The last day of the conference had free papers and PESI-IAPS video presentations by residents. It was very motivating to see that lots of papers were from new institutions and the high quality of papers raised the bar for future PESI-IAPS events.

Kirtikumar J Rathod

PESI-IAPS Secretary 


\section{Gastrointestinal session}

1.

\section{Enteric duplication cyst presenting in late childhood a single center experience}

\section{Rohit Kapoor, Vijai Datta Upadhyaya, Shyamendra Sharma, K. Pujana and Basant Kumar}

\section{Sanjay Gandhi Postgraduate Institute of Medical Sciences, Lucknow}

Background and aim: Enteric duplication (ED) is defined as a congenital lesion that has an anatomical relationship with some part of the bowel is lined by the intestinal mucosa and has a well-developed coat of smooth muscle. They consist of foregut duplication cysts, small bowel duplication cysts, and large bowel duplication cysts. This manuscript deals with small bowel duplication cyst presenting in older children.

Material and methods: An observational cohort study was conducted of 11 patients in single unit between January 2011 and Dec 2020. Children with ED were identified by an electronic record database and operation records. The hospital outpatient records, operation notes, radiological investigations, pathology reports, and discharge summaries were reviewed. Based on hospital data the families were contacted by phone or followed up in the outpatient department.

Results: Eleven EDs in 11 children were identified during the study period of which 7 were male (64\%). Most common presentation was abdominal pain $72 \%$ followed by blood in stool $64 \%$ and recurrent vomiting in $36 \%$ cases. Communication with native bowel was seen in 54\% cases. Laparoscopic assisted excision of the duplicated bowel was done in $45 \%$ case and resection of the bowel along with duplication cyst was required in 63\% (7/11) cases. Ectopic gastric epithelium was observed in $63 \%$ cases and ulceration in the duplicated bowel was seen in $27 \%$ cases.

Conclusions: Ileal duplication cyst may present with occasional bleeding per rectum or anemia hence in older children and should be considered and should be considered as differential diagnosis of such cases.

2.

\section{Laparoscopic Nissen's fundoplication for gastro- esophageal reflux disease and recurrent aspiration pneumonia with laparoscopy assisted feeding gastrostomy in neurologically impaired child additionally-our experience of 3 cases}

\section{Chiranjiv Kumar, Meera Luthra and Apurva Arora}

\author{
Department of Pediatric Surgery, Maulana Azad Medical \\ College, New Delhi
}

Background and aim: To evaluate the role of laparoscopic Nissen's fundoplication for gastro-esophageal reflux disease (GERD) and recurrent aspiration pneumonia with laparoscopy assisted feeding gastrostomy in neurologically impaired child additionally.

Materials and methods: We are presenting 3 cases of GERD and recurrent aspiration pneumonia with seizure disorders (case 1 and 2) or without seizure disorders [case 3]. After radiological assessment with chest $\mathrm{x}$-ray, Gastrograffin swallow and gastro-esophageal reflux scan and pediatric neurology clearance in neurologically impaired patients, they underwent Laparoscopic Nissen's fundoplication with or without laparoscopy assisted feeding gastrostomy. Surgical steps are being demonstrated in the operative video. Course in hospital and condition at the time of discharge are discussed. Pre-operative and post-operative repeat chest $\mathrm{x}$-rays and Gastrograffin swallow are compared and follow-up course discussed.

Results: All 3 cases were kept in Pediatric Intensive Care Unit for $24 \mathrm{~h}$ postoperatively for observation. Foleys catheter removed on postoperative day (POD)1, Nasogastric tube removed on POD3 and abdominal drain removed on POD4. Liquid diet \pm gastrostomy feed started from POD3 followed by soft diet. Sutures were usually removed on POD7. In case 1, F.G. Foleys catheter was replaced by Mickey G tube under screening fluoroscopy on POD14. Post-operative Gastrograffin study in all cases shows no evidence of GER. In all 3 cases, chest conditions improved following surgery both clinically and on repeat chest $\mathrm{x}$-rays.

Conclusions: We must consider laparoscopic Nissen's fundoplication for GERD and recurrent aspiration pneumonia with laparoscopy assisted feeding gastrostomy in neurologically impaired child additionally, as an option who failed to improve on conservative treatment.

\section{3.}

\section{"Pancreas"-an enigmatic organ as always: An eventful pancreatic necrosis sequalae.}

\section{Gandlaparthi Harica Reddy and Prakash Agarwal}

Department of Pediatric Surgery Sri Ramachandra Institute of Higher Education and Research, Chennai

Case presentation: Acute pancreatitis is an emerging problem with an incidence between 3.6 and 13.2 cases/100,000 children. However, necrotizing pancreatitis (necrosis greater than $30 \%$ of the pancreas and/or greater than $3 \mathrm{~cm}$ in an area of the pancreas) is a rare condition ( $<1 \%$ of acute pancreatitis), with a presentation similar to uncomplicated pancreatitis cases and with high morbidity and mortality. Computed tomography (CT) allows an assessment of the severity of the disease and the risk of complications (CT Severity Index-CTSI). Uncomplicated pancreatitis cases have a favourable outcome, but necrotizing pancreatitis cases require intensive medical treatment and surgical treatment (minimally invasive necrosectomy). We report the case of an 11-year-old girl with clinical presentation compatible with acute pancreatitis and confirmed by abdominal ultrasonography. Due to worsening of symptoms, an abdominal computed tomography was performed showing a necrotizing pancreatitis and large walled off necrosis for which minimally invasive necrosectomy is done. Later after 3 weeks she developed pancreatic abscess which was managed by minimally invasive surgery. Close monitoring and an aggressive approach were adopted, with clinical and analytical improvement. The paediatric necrotizing pancreatitis is an uncommon and potentially severe entity; we must keep this complication in mind for an adequate differential diagnosis of acute abdomen. 
4.

\section{Laparoscopic management of isolated jejunal Chron's stricture in a child}

\author{
Sayak Chattopadhyay and Prakash Agarwal
}

\section{Department of Pediatric Surgery, Sri Ramachandra Institute} of Higher Education and Research, Chennai

Background and aim: Isolated jejunal Crohn's disease is quite a rare finding representing approximately $1 \%$ of cases in adults. In fact, there is a dearth of literature on prevalence of the same in children. Here we aim to highlight a multidisciplinary approach in tackling the condition and the use of laparoscopy assisted technique in the management of the stricturous obstruction.

Materials and methods: A 13-year-old male presented with multiple episodes of bilious vomiting. The patient had been diagnosed by the paediatric gastroenterologist as a case of Crohn's disease 2 years before. He relapsed one month back and was started on Azathioprine and Adalimumab therapy. Vomiting however persisted. An esophagogastroduodenoscopy and a colonoscopy with biopsy showed no signs of inflammatory bowel disease.

Results: The paediatric surgeon performed a diagnostic laparoscopy revealing a hugely dilated jejunum with oedematous walls proximally followed by a $15 \mathrm{~cm}$ segment of thick stricturous jejunum and distal collapsed ileum. A bowel walk ruled out the presence of any skip lesions. Hence, they proceeded with a laparoscopy assisted resection of the stricturous segment with extracorporeal end to end anastomosis. The patient is currently symptom free 2 months post surgery.

Conclusions: To sum it up this is a rare case of isolated jejunal Crohn's which underwent a swift resolution due to active interaction between gastroenterology and paediatric surgery consultants. Also, in a scenario where the patient has anaemia, low serum albumin levels and growth failure, a laparoscopic approach with extension of the umbilical port incision for specimen retrieval vastly overshadows a laparotomy in terms of morbidity and wound healing.

\section{5.}

\section{Laparoscopic versus open appendicectomy for complicated appendicitis in children: A systematic review and meta-analysis}

\section{Arka Banerjee, Sujoy Neogi, Shasanka S. Panda, Simmi K. Ratan and Raghav Narang}

\section{Department of Pediatric Surgery, Maulana Azad Medical College, New Delhi}

Background and aim: The aim of this meta-analysis was to comprehensively review the current literature regarding the various postoperative outcomes after laparoscopic appendicectomy (LA) and open appendicectomy (OA) in order to determine the best approach for children with complicated appendicitis (CA).

Materials and methods: The PRISMA guidelines were adhered to and an extensive electronic database search was performed. Data analysis, including subgroup analysis of randomized-control trials, was performed using RevMan 5.3. Methodological and statistical heterogeneity, as well as publication bias of the included studies, were assessed.

Results: Four randomized controlled trials (266 LA versus $354 \mathrm{OA}$ ) and thirty six case-controlled trials (2580 LA versus 3043 OA) were included in the analysis. Compared to OA, LA was found to have a shorter length of stay, a lower rate of surgical site infection as well as a significantly lower overall complication rate. The rates of intraabdominal abscess formation, post-operative fever, pneumonia, ileus, r-admissions and re-operations were similar in the two groups. LA was also found to have a shorter time taken to oral intake and a lesser requirement of analgesics as well as intravenous antibiotics. Operative time for OA was found to be significantly shorter than that for LA.

Conclusions: The main concern with LA was the higher rate of intraabdominal abscess in patients with $\mathrm{CA}$ but this meta-analysis clearly shows that the incidence rates are similar even after OA. Hence, together with other improved postoperative outcomes, LA should be the procedure of choice in children with CA.

\section{6.}

\section{Role of diagnostic laparoscopy in successful management of extraperitoneal penetrating rectal injury}

\section{Dhruv Mahajan, D. Hari Krishna, Devedra Kumar Yadav, Prabudh Goel and Minu Bajpai}

Department of Pediatric Surgery, All India Institute of Medical Sciences, New Delhi

Background and aim: Rectal injuries are an uncommonly encountered entity in children. Though rarely seen in clinical practice, the significance of adequately managing these injuries lies in the otherwise high mortality and morbidity. Historically, primary repair with or without diversion, rectal washouts and presacral drainage were the preferred therapeutic interventions. The aim of this presentation is to describe a case of penetrating rectal perforation successfully managed by a diagnostic laparoscopy followed by conservative management. Materials and methods: An 8-year-old boy presented with an accidental penetrating injury by a sharpened pencil $8 \mathrm{~cm}$ away from the right anal margin with complaints of pain abdomen. Computed tomography pelvis revealed mild free fluid in pelvis with multiple air pockets were in pelvis. A foreign body of length around $9 \mathrm{~cm}$ was noted lying inside the lumen of rectum with the proximal end just reaching up to sigmoid colon.

Results: The foreign body was removed en masse on per rectal examination and diagnostic laparoscopy was done in view of suspected peritoneal contamination. No intraperitoneal bowel injury, pelvic collection or inflammation was seen. A retroperitoneal hematoma, just to the right of rectum was seen. Child was managed conservatively without colostomy. Oral feeding was started after 3 days and child had unremarkable recovery.

Conclusions: Diagnostic laparoscopy is a useful tool for intraperitoneal inspection and can help to rule out any peritoneal contamination and should the first step, negating stoma creation. It helps to avoid morbidity associated with open exploration. A diversion colostomy is not required in all patients with extraperitoneal rectosigmoid perforation. 
7.

\section{Laparoscopy assisted management of mesenteric cyst in children: case series}

Sayak Chattopadhyay and Prakash Agarwal

Department of Pediatric Surgery, Sri Ramachandra Institute of Higher Education and Research, Chennai

Background and aim: In this study we aim to highlight the importance of diagnostic laparoscopy in planning the approach to a mesenteric cyst. This coupled with a laparoscopy assisted excision or resection anastomosis as required pave the way for faster recovery and minimal post-operative morbidity.

Materials and methods: A total of 10 cases diagnosed with mesenteric cyst in the Department of Paediatric Surgery in Sri Ramachandra Institute of Higher Education in the last 2 years are included in this study.

Results: Out of 10 cases, 6 were diagnosed by ultrasonography of whole abdomen, 3 were diagnosed by computed tomography (CT) of whole abdomen while one was diagnosed on magnetic resonance imaging (MRI). 8 patients presented with vague abdominal pain, 6 had palpable mass per abdomen while 4 presented with vomiting. All patients underwent diagnostic laparoscopy followed by laparoscopy assisted approach. 1 case was converted to open. 7 cases underwent excision of cyst with resection and anastomosis while 3 cases underwent excision alone. Intraoperatively 6 cases were found to involve the jejunal mesentery, 2 were found to involve the ileum while 1 was found adherent to second part of duodenum and 1 in the subhepatic region. Postoperatively patient was started on feeds on post-operative day 3 on an average. Total mean hospital stay was 5 days. Patients were followed up for one year and showed no recurrence or morbidity.

Conclusions: Laparoscopic assisted approach not only helps to triangulate the exact location of the mesenteric cysts, it also leads to an earlier recovery and shorter hospital stay in children.

8.

\section{Laparoscopic modified Swenson's procedure for Hirschsprung's disease: technical considerations and initial experience}

\section{Ankur Mandelia, Shyamendra Pratap Sharma, Rohit Kapoor and K. Pujana}

\section{Department of Pediatric Surgery, Sanjay Gandhi Postgraduate} Institute of Medical Sciences, Lucknow

Background and aim: To describe our technique and initial experience with laparoscopic modified Swenson's procedure (LmSw), which combines the advantages of laparoscopic rectal mobilization and transanal perineal dissection.

Materials and methods: From July 2019 to February 2021, 12 children with biopsy-proven Hirschsprung's disease (HD) underwent LmSw. Patients were placed in lithotomy position and three ports were used. The proximal ganglionated bowel was mobilized, and distal circumferential rectal dissection was continued below the peritoneal reflection, down to the pelvic floor. The perineal part of the procedure started with placement of anal retraction sutures.
Interrupted stay sutures were placed in the rectal mucosa $1 \mathrm{~cm}$ above the dentate line. A full-thickness circumferential rectal incision was made and dissection was performed close to the rectal wall till it joined with the laparoscopic plane of dissection. The recto-sigmoid was then withdrawn, avoiding twisting of the pull-through segment. The colo-anal anastomosis was fashioned in two layers using absorbable sutures.

Results: Median age and weight at operation was 3 years and $14 \mathrm{~kg}$, respectively. 9 patients with stoma underwent a three stage procedure and three patients underwent a primary pull through. 9 out of 12 patients had classical recto-sigmoid HD. 1 patient had total colonic aganglionosis (TCA) and required conversion to open. The median operating time was $210 \mathrm{~min}$ and median length of resected bowel was $18 \mathrm{~cm}$. The median time to full feeds and hospital stay was $36 \mathrm{~h}$ and 5 days, respectively. Median follow up duration is 8 months. 1 patient had post-operative enterocolitis which was managed conservatively. 8 patients have attained normal stooling pattern with no fecal or urinary incontinence. 1 patient required occasional rectal washouts for intermittent constipation. 3 patients are awaiting stoma closure.

Conclusions: $\mathrm{LmSw}$ is a feasible, safe and effective procedure for the laparoscopic management of HD in children with acceptable short term results

Declaration: Part of the data was presented at International Pediatric Colorectal Congress in November, 2020. This presentation has updated data and video.

9.

\section{Laparoscopic hernia repair in can prevent development of patent process vaginalis to develop in hernia}

\section{Shyamendra Sharma, Vijay Datta Upadhyaya, \\ Basant Kumar, K. Pujana and Rohit Kapoor}

Department of Pediatric Surgery, Sanjay Gandhi Postgraduate Institute of Medical Sciences, Lucknow

Background and aim: Inguinal hernia repair is the second-most commonly performed operation in pediatric patients. In young pediatric patients, these repairs are typically performed with a high ligation of the hernia sac, either by open technique or laparoscopically. Usually in open technique hernia is repaired in the side which it is evident and these patients some time present with clinically evident or symptomatic hernia of other side. We are presenting our pilot series of laparoscopic hernia repair were processes vaginalis was patent on contra-lateral side of clinically evident hernia.

Materials and methods: All the cases of hernia aged above 3 years admitted in last one year in single unit were included in the study. The data regarding the side of the hernia, age, sex and method of repair were retrieved from electronic record system of hospital. Open or laparoscopic hernia repair was done on the demand of the patient's parent. In all cases the record regarding consent for open or laparoscopic repair was checked from record.

Results: A total of 25 new cases of inguinal hernia aged above 3 years were managed during this period. Most of the cases were repaired with open technique and only seven cases were repaired laparoscopically. Among the open repair two out of 18 cases were readmitted for development of clinical or symptomatic hernia on contralateral site. Among seven cases operated laparoscopically had patent processus vaginalis (PPV) on contralateral side which was ligated after obtaining due consent. In none of patients recurrence of hernia was observed. 
Conclusions: Laparoscopic hernial repair is safe in children and can help to minimize the chances of dormant PPV to develop into clinical/ symptomatic hernia. Though large series is needed to reach any definitive conclusion.

10.

\section{Pediatric laparoscopic hernia repair: a single-institute experience}

\section{Rashmi and Vijay Kumar Kundal}

\section{Department of Pediatric Surgery, Dr. Ram Manohar Lohia Hospital, New Delhi}

Background and aim: Open herniotomy with high ligation is considered gold standard in the management of pediatric inguinal hernia. Although laparoscopic hernia repair has emerged as a superior alternative in terms of cosmesis and approach to opposite side, speculations regarding the increased recurrence rates and prolonged operative time prevent it from being accepted as a standard of care. Materials and methods: A restrospective study was conducted on all patients who underwent laparoscopic hernia repair in the Department of Pediatric Surgery, Dr. RML hospital, New Delhi, during the period of Jan 2016-Dec 2020. All the case records were studied with respect to age, sex, site of hernia, duration of surgery and post-operative complications.

Results: A total of 24 patients underwent laparoscopic hernia repair during the study period. $25 \%(n=6)$ patients were females and the rest $(\mathrm{n}=18)$ were males, the male to female ratio was $3: 1$. The average age at presentation was 5.4 years (range $1-12$ years). In $66.6 \%(\mathrm{n}=16)$ patients right herniotomy was done, while $20.8 \%$ $(\mathrm{n}=5)$ patients underwent left herniotomy. In $12.5 \%(\mathrm{n}=3)$ patients, bilateral hernia repair was performed. In 1 patient, conversion to open herniotomy was done. The average duration of surgery was 48 min. Postoperatively, 2 patients developed scrotal edema. No other complications (including recurrence) were noted.

Conclusion: Laparoscopic inguinal hernia repair can be offered as a standard of care as it is safe, reproducible, technically easy and cosmetically appealing. It also offers better accessibility in $\mathrm{B} / \mathrm{L}$ inguinal hernia repair.

\section{Karl Storz session}

\section{1.}

\section{Laparoscopic hepatico-jejunostomy for choledochal cysts with aberrant right hepatic artery: technical considerations and initial experience}

\section{Rohit Kapoor, Ankur Mandelia, Ashwani Mishra, Shyamendra Sharma and K. Pujana}

\section{Department of Pediatric Surgery, Sanjay Gandhi Postgraduate Institute of Medical Sciences, Lucknow}

Background and aim: Vascular anomalies at the hepatic hilum present a technical challenge in laparoscopic management of choledochal cysts (CDC). The aim of this study was to describe our surgical approach in identification and management of aberrant right hepatic artery (RHA) during laparoscopic hepatico-jejunostomy (HJ) for CDC.

Materials and methods: From November, 2019 to February, 2021, 11 children with Type 1 CDC underwent laparoscopic excision of CDC with HJ. Aberrant RHA was recognized in three cases (27.3\%) upon initial inspection and dissection of Calot's triangle. Following dissection of the cyst and transection of the CHD at an appropriate level, the RHA was carefully dissected off the anterior wall of the CHD and transposed posterior to the CHD. Hepatico-jejunostomy was fashioned intracorporeally, anterior to the posteriorly transposed RHA.

Results: Mean age and weight at operation $(n=11)$ was 4 years and $18 \mathrm{~kg}$, respectively. The mean operating time was $360 \pm 60 \mathrm{~min}$. Conversion to open surgery was required in $2(18.2 \%)$ patients. The mean time to full feeds and hospital stay was $80 \pm 12 \mathrm{~h}$ and $7 \pm 1.5$ days, respectively. Minor bile leak occurred in $1(8.3 \%)$ patient which resolved with conservative management on post-op day 3 . None of the study parameters were significantly different in patients with aberrant RHA $(n=3)$ compared to those with normal vascular anatomy $(\mathrm{n}=8)(\mathrm{p}>0.05)$. All patients have normal liver function tests and satisfactory ultrasonogram and hepatobiliary scintigraphy reports at last follow-up.

Conclusions: Our experience demonstrates that repositioning the RHA posterior to the CHD during laparoscopic surgery for CDC is technically feasible and safe. This is essential not only to restore normal anatomy and to create adequate space for $\mathrm{HJ}$, it is also important to prevent recurrent biliary obstruction and subsequent hepatocellular damage.

12.

\section{Retroperitoneoscopic Uretero-ureterostomy for Retrocaval Ureter}

Deepti Pai and Ravi Prakash Kanojia

\section{Department of Pediatric Surgery, Postgraduate Institute of Medical Education and Research, Chandigarh}

Background and aim: Retrocaval Ureter (RCU) is a condition which makes the right ureter to abnormally course around the IVC. This leads to upper ureteric obstruction and warrants surgical correction. The surgical methods involves delicate handling of both the ureter and IVC. The condition can be approached both trans-peritoneally (TP) and retroperitoneally. The presented video demonstrates the retroperitoneoscopic (RP) approach to RCU showing excellent visualisation during corrective surgery. Various pros and cons of RP and TP approaches for this condition are discussed.

Materials and methods: A 10-year old male presented with pain abdomen for 2 years. Ultrasound showed presence of right hydronephrosis with upper ureteric dilatation. Computed tomography (CT) urography showed fishhook appearance on right side suggestive of RCU. Child was taken up for RP right ureteroureterostomy. With patient in left lateral position, a camera port and two working ports were placed in right lumbar region via the posterior abdominal wall to enter retroperitoneal space. Ureter was mobilised and divided at medial border of inferior vena cava. After untangling it, ureteroureterostomy was performed in an end-to-end fashion over double $\mathrm{J}$ ureteric stent (DJ stent). 
Results: Child was discharged after $36 \mathrm{~h}$ and had an uneventful stay. DJ stent was removed after 4 weeks. Child remained asymptomatic in follow up period. Renal dynamic scan (Tc-99 m ethylene dicysteine scan) showed unobstructed drainage of tracer on right side.

Conclusions: RCU is a rare congenital anomaly. RP approach for the treatment of RCU gives an advantage of excellent exposure, cosmetic results and less post-operative ileus. There is no requirement of extensive mobilisation of right colon, which is otherwise needed in a transperitoneal approach. Access to retroperitoneum is technically challenging factor in pediatric age group and may be a limiting factor while making the choice by the surgeon.

Declaration: This abstract has not been presented at another scientific meeting. Part of this work has been published as clinical images in Journal of Indian Association of Pediatric Surgeons in 2017, 22nd edition, "Retrocaval Ureter: Clinical Images".

\section{3.}

\section{Seldinger technique in easy port placement for Pneumovesicum (STEPP)-A novel, safe and fail- proof method}

\section{Apurva Arora, Bikash Kumar Naredi and Bibekanand Jindal}

\section{Department of Pediatric Surgery, Jawaharlal Institute of Postgraduate Medical Education and Research, Puducherry}

Background and aim: We showcase our novel improvisation in port placement method for creating pneumovesicum for vesicoscopic ureteral reimplantation for primary vesico-ureteric reflux in children. Materials and methods: We have retrospectively studied the time taken for port placement, port related failure and complication rates, port related- conversion to open- in our initial eight cases of vesicoscopic ureteral reimplantation with the Mohan Abraham urethral sound railroading technique, with our last four cases where we have tried our novel Seldinger Technique in Easy Port Placement (STEPP) method for port placement.

Results: We have reviewed our series of 12 vesicoscopic bilateral cross-trigonal reimplantation-analogous to open Cohen's ureteric reimplantation in the last five years. Mean patient age-5.3 years. Mean operating time-378 min. Port related issue like port displacement and air leak in six cases which operated by the urethral sound railroad technique in our initial cases. No air leak and port displacement or port related events occurred during surgery in last four cases operated with our STEPP technique, thereby reducing the overall operating time and the surgeon's stress. None of the patients required conversion to open. Maximum follow up is 5 years and minimum is 6 weeks, with resolution of reflux near 100\% (last 3 cases are due to postop DRCG).

Conclusions: We showcase our STEPPP technique for port placement for creating pneumovesicum, in addition to visual confirmation with a cystoscopy, its fast, safe, accurate and leak proof. Our novel technique is easy to replicate and has minimal intra-operative complications, we recommend this for port placement for vesicoscopy.
14.

\section{Sestemibi guided thoracoscopic excision of functional mediastinal parathyroid adenoma}

\author{
Rahul Dey, Abhishek Reddy, A. Q. Nabeel, M. M. Zameer, \\ C. Vinay, Sanjay Rao and Ashley D'Cruz
}

Department of Pediatric Surgery, Narayana Health, Bangalore

Background and aim: To describe the management of a child with an ectopic parathyroid adenoma using thoracoscopic surgery.

Materials and methods: A 12 years old girl presented with the chief complaints of difficulty in walking with progressive lower limb deformity and a history of frequent fractures in the upper limbs after trivial trauma. X rays of the hand and skull showed decreased bone density. Blood investigations revealed increased levels of calcium, PTH and ALP. Sestamibi scan showed retention of isotope in the retrosternal area. Contrast enhanced computed tomography (CECT) neck was done to know the extent and vascular anatomy of the lesion. Results: The child underwent thoracoscopic surgery and excision of tumour, under radioisotope navigation.. General anaesthesia with single lung (right) ventilation was done for ease of procedure. Intraoperative PTH levels and frozen section were used to ensure complete resection. Child was extubated on table. Drain was removed on post op day 3. Serial monitoring showed a downward trend of serum PTH levels. Histopathology was confirmatory of an intra-thymic parathyroid adenoma. Child was discharged on post op day 4 .

Conclusions: VATS and excision of the tumour is a minimally invasive and effective treatment modality for children with ectopic parathyroid adenomas. Sestamibi scan and computed tomograpy/magnetic resonance imaging are key to success by providing pre-operative localisation of the lesion.

\section{5.}

\section{Thoracoscopic repair of iatrogenic bronchial injury in a neonate during bronchogenic cyst excision}

\section{Revathy Menon, Kirtikumar Rathod, T. K. Jayakumar, Prawin Kumar, Daisy Khera, Manoj Kamal and Arvind Sinha}

Department of Pediatric Surgery, All India Institute of Medical Sciences, Jodhpur

Case presentation: Our patient is a $3.6 \mathrm{~kg}$ male diagnosed with severe tracheobronchomalacia referred from the neonatal intensive care unit (NICU) for management of a cystic mediastinal lesion. Child was taken up for thoracoscopic excision of the cyst. The infant thoracic cavity is a challenging surgical field for thoracoscopic approach since it is a rigid small working space with structures continuously in motion. Difficult anatomy due to the abnormal lesion also poses a significant challenge during the surgery. We identified a bulging cyst behind the esophagus in mediastinal pleura in close proximity to azygous. Special attention is to be paid so as to not injure the adjacent structures such as the esophagus, azygous or bronchus. During dissection with hook cautery, there was accidental injury to the left bronchus. ET $\mathrm{CO}_{2}$ was rapidly increasing since we were insufflating his airway with our $\mathrm{CO}_{2}$. This is a good way to identify 
airway injury during thoracoscopic surgery. Using prolene 5-0 suture, the rent was meticulously sutured. Our anesthetist were quite supportive and they stopped ventilating the baby intermittently as we were repairing the bronchus. To confirm the absence of any leak, the anesthetist was requested to ventilate the injured bronchus and no bubbling signified a properly sealed repair. Post operatively patient was kept on ventilator in view of post-operative tracheobronchomalacia which improved gradually. He was weaned off ventilator by post-opreative day (POD) 5 and maintained saturation at room air by POD 8. Patient tolerated full feeds, gained adequate weight and was discharged by POD 15 .

Conclusions: A timely intervention with the help from a dedicated and capable anesthesia team have proven life saving in our patient and avoided conversion to open. Operative duration was around $2 \mathrm{~h}$. Despite facing such complications, our experience suggests that we can opt to tackle this in a minimally invasive technique.

\section{6.}

Total laparoscopic gastric pull up in a case of corrosive esophageal injury with stricture

\author{
Saji Vargheese, Thirugnanasambandam Nelson, \\ Shiv Kumar, Joash Jensen and Anis Akhtarkhavari
}

Department of Pediatric Surgery, G.B. Pant Hospital, Port Blair

Background and aim: Corrosive esophageal injuries in children are commonly caused due to unintentional ingestion. Severe injuries can result in dense and long esophageal strictures, where esophageal replacement remains the lone option. Minimally invasive surgery for esophageal substitution is a novel and less morbid approach performed at very limited centers worldwide in children. Corrosive induced adhesions and scarring make laparoscopic esophageal replacement an unnerving surgery.

Materials and methods: We present an 11-year-old boy with accidental ingestion of acid, which was initially managed conservatively. He developed esophageal stricture leading to persistent vomiting and failure to thrive. On evaluation child had a long segment stricture involving the lower and middle 1/3rd of his esophagus, not amenable to endoscopic dilatations. A laparoscopic trans hiatal esophagectomy with gastric pull up with cervico-esophagogastric anastomosis with pyloroplasty and feeding jejunostomy was performed.

Results: Apart from bleeding through the trans anasotomotic nasogastric tube which was managed conservatively, the child had an early and uneventful recovery. Was started on liquids on post-operative day 8 after a satisfactory barium swallow. Feeds were gradually progressed to semisolids. He was discharged on post-operative day 10. On 1 year follow up the child is thriving well, tolerating normal feeds and is on no medications.

Conclusions: With limited working space and tarnished mediastinum in corrosive esophageal injury in children, minimally invasive surgery for esophageal replacement is a very colossal task. Nevertheless, when done by competent hands with advanced laparoscopic skills in the company of experienced support team, it produces excellent results with considerable decrease in postoperative morbidity.

\section{Short video session}

17.

\begin{abstract}
A novel technique of combined posterior sagittal anorectoplasty with laparoscopy for management of anorectal malformation: our experience
\end{abstract}

\section{Mohammad Imran and Vaibhav Pandey}

Department of Pediatric Surgery, Institute of Medical Sciences, Banaras Hindu University, Varanasi

Background and aim: Posterior sagittal anorectoplasty (PSARP) is the gold standard for management of ARM. Laparoscopic assisted anorectoplasty (LAARP) has shown equally good results. We present our novel technique of combined PSARP with laparoscopy for management of anorectal malformation(ARM).

Materials and methods: The study was conducted between January 2018 and Febuary 2021 including all the patients $(n=3)$ with rectovesical fistula who underwent surgery and managed by combined PSARP with laparoscopy. The operative technique was as follows. Under general anesthesia, the baby is placed in a prone position with the buttock raised high with bolster. A posterior sagittal incision made and mid-point of anal sphincter identified and a $12 \mathrm{~mm}$ trocar placed and fixed at the site as anal opening to be made. Patient turned supine and a $10 \mathrm{~mm}$ camera port is placed via a supraumbilical incision. Two instrument ports $(5 \mathrm{~mm})$ are placed at right and left lower quadrants of the abdomen. The peritoneum covering the rectum is opened up and dissected circumferentially towards the distal fistula. When the most distal end of the fistula where it inserts into the genitourinary tract is exposed, it is divided. Under direct visualization, the distal rectum is retrieved via the $12 \mathrm{~mm}$ anal trocar and anoplasty done.

Results: The short term and mid term outcome of our technique were comparable to Abdomino PSARP. With our combined technique there was lesser post operative pain, lesser operative blood loss and better cosmetic outcome.

Conclusions: Combined PSARP and laparoscopy has results comparable to PSARP approach with better outcomes in rectovesical fistula.

18.

\section{Laparoscopic duodenal web excision and duodeno- duodenostomy in a girl previously treated for malrotation: A missed opportunity}

\section{Manasa Reddy and Ravi P. Kanojia}

\section{Department of Pediatric Surgery, Postgraduate Institute} of Medical Education and Research, Chandigarh

Background and aim: Duodenal web often presents as chronic duodenal obstruction and may have a delayed presentation with significant morbidity to the patient. Diagnosis can be established by upper GI contrast study and laparoscopic management provides an effective way of excising the web and duodenal repair. Identifying the correct duodenal-otomy site is sometimes tricky and needs to be 
identified by some surface markers. The presented video shows duodenal web excision and repair with some details on handling the duodenum during the procedure.

Materials and methods: A 6-year-old female child underwent laparoscopic Ladd's procedure for malrotation at 8 months of age. She continued to have intermittent symptoms of non-projectile vomiting, mainly on taking solid food. On examination, vital signs were normal and fullness was noted in the epigastrium. There was no evidence of visible gastric peristalsis. UGI contrast study revealed massively dilated D1 and D2 with slit like narrow pinpoint opening in D2. She was taken up for laparoscopic exploration. With $10 \mathrm{~mm}$ optical port in left iliac region and two $5 \mathrm{~mm}$ working ports in left lumbar and right iliac region, duodenum was kocherised. Transition zone was noted at D2-D3 junction. Thick fleshy web was excised in its lateral part after duodenotomy. Heineke Mikulicz type repair of duodenum was done over 12 Fr nasojejunal tube.

Results: Child was started on nasojejunal feeds on POD 1 and allowed orally on sixth post-operative day. She was discharged on post-opreative (POD) 7 and remained asymptomatic on follow up after 1.5 years.

Conclusions: Duodenal web is a variable component of anomaly of malrotation and can be missed during Ladd's correction of malrotation. Continued symptoms should prompt for possibility of missed web. It's quite feasible to approach a previously operated abdomen by open surgery by laparoscopic approach. Accurately identifying the site of duodenotomy is important so that a long otomy of duodenal wall is avoided. The video presentation demonstrates the procedure with all the technical details.

\section{9}

\section{Supraglottoplasty—an endoscopic management of laryngomalacia with laryngeal micro-scissors}

\author{
Sai Lavanya, A. Q. Nabeel, M. M. Zameer, C. Vinay, \\ Sanjay Rao and Ashley D'Cruz
}

\section{Department of Pediatric Surgery, Narayana Health Multispecialty Hospital, Bangalore}

Case presentation: To describe the procedure of supraglottoplasty in the management of severe laryngomalacia. 2 months old male baby presented with inspiratory stridor and respiratory distress with sub sternal retractions. He was evaluated with micro-laryngo bronchoscopy in view of severe laryngomalacia.

On micro-laryngo bronchoscopy, child was found to have the following findings:

- Omega shaped epiglottis

- Shortened bilateral aryepiglottic folds

- Redundant arytenoid mucosa on right side

- Collapse of arytenoid complex during inspiration

- Normal movement of vocal cords

Supraglottoplasty was done with minimal tissue damage using micro-laryngeal scissors and laryngeal grasper. Stridor was resolved and child was comfortable post operatively.

Conclusions: Ablation of shortened aryepiglottic fold led to enlargement of Laryngeal inlet and improvement of upper airway. So, supraglottoplasty remains an effective method and can avoid tracheostomy in the surgical management of severe laryngomalacia.
20.

Auto-amputated ovarian cyst—a unique finding

Somya Bhatt, Rahul Saxena, Arvind Sinha, Manish Pathak, Kirtikumar Rathod and Avinash Jadhav

Department of Pediatric Surgery, All India Institute of Medical Sciences, Jodhpur

Case presentation: Ovarian cystic lesions are commonly dealt with in paediatric surgery. sThese cysts can be functional ovarian cyst, benign tumours or even malignant neoplasm in nature. Therefore, while it is essential to make a distinct differential diagnosis it is however difficult to make so due to various reasons such as the disturbance created by other abdominal masses, mobility of the cyst itself etc.

The current case study describes the clinical case of a 5-month-old baby who showed the presence of a mass between the kidney and the ovary during antenatal USG. The mass was well-defined and measures $4 \times 3.5 \times 4.5 \mathrm{~cm}(\mathrm{AP} \times \mathrm{TR} \times \mathrm{CC})$ rising from the ovary and extended to the pelvis midline posterior to the urinary bladder. Overall, it was a well-defined cystic lesion with heterogenous internal contents in the right ovary. Hence the diagnosis was made to be ovarian dermoid cyst.

Based on the diagnosis laparoscopic excision of the ovarian cyst was conducted. However, during the surgery it was noticed that the mass was in fact a chocolate brown cyst which was autoamputated. The results obtained during the surgery changed the diagnosis to a self-amputated cyst in an infant making this case report unique in nature. The results were later also confirmed by biopsy which categorised the mass as benign ovarian cyst. This case report not only highlights the difficulties in diagnosis of ovarian cyst but also presents a unique case of self-amputated cyst and its management.

21.

\section{Port exteriorization appendectomy in children: our experience}

\author{
Manika Boipai, Rahul Gupta, Bhairu Gurjar, \\ Priyanka Mittal and Praveen Mathur
}

\section{Department of Pediatric Surgery, SMS Medical College, Jaipur}

Background and aim: To describe our experience using the port exteriorization appendectomy and assess its safety, efficacy and feasibility in children with appendicitis.

Materials and methods: We retrospectively studied 20 patients (M:F = 4:1) who had port exteriorization appendectomy between January 2017 and February 2021. The patients with generalized peritonitis were excluded. All patients had their laparoscopic appendectomy within $48 \mathrm{~h}$ of admission. The procedures were done by consultants or M.Ch. residents under complete supervision of consultants (with experience in minimally invasive techniques). Appendectomy was performed extra-abdominally (extra-corporally) after the appendix was identified by using a laparoscope through $5 \mathrm{~mm}$ umbilical optical port and then delivered outside through the $10 / 12 \mathrm{~mm}$ working port (second port) in right iliac fossa. Technical challenges, complications, and postoperative recovery data were collected and analyzed. 
Results: 20 Children with a mean age of 9.9 years (6-15 years) underwent laparoscopic appendectomy using the port exteriorization technique. In $30 \%$ of the cases laparoscopic appendectomy were performed in complicated appendicitis (lump/local collection); the accessory port was required in in these patients $(30 \%)$ to complete the appendectomy. No major complications were reported. The mean hospital stay was 4.3 days (3-6 days). Postoperative recovery and cosmetic results were excellent in all cases. The port site infection was seen in $5 \%$ (1) patient.

Conclusions: Port exteriorization appendectomy is a safe and economical approach in pediatric patients, when conditions are favorable. It can be performed by M.Ch. residents in the beginning of their learning curve with laparoscopic surgery. It allows a low level of invasiveness with satisfactory cosmetic results. The overall morbidity was low.

22.

\section{Per urethral non optical forceps: a convenient aid during vesicoscopic ureteric reimplantation}

\author{
Nellai Krishnan Subramonian, Vishesh Jain, \\ Anjan Kumar Dhua, Devendra Yadav, Prabudh Goel, \\ Sandeep Agarwala and Minu Bajpai
}

\section{Department of Pediatric Surgery, All India Institute of Medical Sciences, New Delhi}

Background and aim: To present a case of vesicoscopic ureteric reimplantation, where a thin non optical forceps passed per urethrally, which was used to hold the ureteral stay suture, aided in easy ureteral dissection.

Materials and methods: A 5 years old male presented with the complaints of abdominal pain of 6 months duration associated with intermittent episodes of Urinary tract infection. On examination, no abdominal lump was palpable. Transabdominal ultrasonography showed left hydroureteronephrosis. Micturating cysto-urethrogram (MCU) was done, which showed Grade III vesicoureteral reflux on left side. Post void residue was insignificant. Decision for vesicoscopic left ureteric reimplanation was made as patients guardians refused endoscopic Deflux treatment. Patient was positioned in lithotomy. Standard port placed with the aid of Clutton's curved urethral dilator. During the procedure, need for additional retractor was felt for optimal traction and countertraction to aid in dissection of ureter. So a thin non optical forceps was passed per urethrally, which was used to hold the ureteral stay suture and aided in easy ureteral dissection.

Results: The surgery was uneventful. Patient was allowed orally on the day of surgery. Post operatively patient was asymptomatic. Urethral catheter was removed after $72 \mathrm{~h}$ and the patient sent home. Micturating cysto-urethrogram (MCU) done 3 months post operatively showed no reflux.

Conclusions: A thin non optical forceps passed per urethrally can be used to hold the ureteral stay suture, which can make the ureteral mobilization easier during vesicoscopic ureteric reimplantation. This technique may particularly useful for novice surgeons.
23.

\section{Minimally invasive management of post traumatic bladder neck fistula}

\section{T. Heera and Ramesh Babu}

Department of Pediatric Surgery, Sri Ramachandra Insitite of Higher Education and Research, Chennai

Background and aim: Pelvic fracture urethral distraction (PFUD) usually leads to stricture warranting endoscopic or open management. We herewith report a rare case of bladder neck fistula managed via minimally invasive approach.

Case presentation: An 8-year-old boy with history of pelvic fracture following a run over by tractor 1 year ago; during initial admission had intra pelvic extravasation with Foley bulb found lying outside the bladder. An open SPC was performed followed by rail roading of per urethral catheter. Six months later he undwent cystoscopy-optical internal urethrotomy for stricture. Following this he was able to void urine in good amount early morning but there was constant daytime dribbling in between the voiding episodes. micturating cystourethrogram (MCU) and computed tomography (CT) urogram revealed bladder neck fistula due to a false passage and this was draining into urethra distal to the sphincter. Vesicoscopic closure of false passage was attempted with 5-0 vicryl. However post procedure dribbling was persistent and a repeat MCU after 4 weeks revealed recurrence of bladder neck fistula. Subsequently, cystoscopic fulguration of mucosa of false passage was performed both antegrade and retrograde fashion. Per urethral catheter was kept for 6 weeks; follow up MCU revealed closure of false passage. Dribbling improved clinically, child is on follow up.

Conclusions: Bulbomembranous urethra stricture is the commonest complication of PFUD. However, intra pelvic extravasation, bladder neck injury leading to false passage of urethra and incontinence is uncommon. This case is being presented for its rarity and to demonstrate the minimally invasive management approach.

24.

\section{Robot-assisted laparoscopic extravesical ureteral reimplantation: a new emerging minimally invasive modality}

\section{Prabhu Karunakaran, Zain Tamboli, Punith Mathur, Abhiskek Shitanshu, Abhay Navneeth, Danish Gourav, Y. Priyank and M. S. Ansari}

\section{Sanjay Gandhi Postgraduate Institute of Medical Sciences, Lucknow}

Case presentation: A 6 years male child with Right single functioning kidney, evaluation for urinary tract infection (UTI) and raised creatinine, investigations like X-ray, ultrasonography, renal scans, magnetic resonance urography (MRU) revealed Right lower ureteric stricture with concurrent multiple renal calculi (probably secondary) and child underwent percutaneous nephrolithotomy (PCNL) followed by robotic right extra vesical ureteric reimplantation after 3 weeks interval. 
Results: Child underwent extra vesicle anterior wall ureteric reimplantion, console time was $150 \mathrm{~min}$, blood loss was $30 \mathrm{ml}$. Postoperative recovery was uneventful, PUC removed after $48 \mathrm{~h}$, DT was removed after $72 \mathrm{~h}$ and Patient was discharged on post-op Day 4. Post op MCU was normal and Renal scan showed non obstructive drainage pattern.

Conclusions: Robotic extravesical ureteric reimplantation is the new minimally invasive modality. The technique is safe ang gives optimum surgical outcome.

\section{5.}

\section{Laparoscopic management of liver and retro-vesical hydatid cyst}

\author{
Manasa Reddy, Nitin J. Peters, Muneer A. Malik, \\ Shubhalaxmi R. Nayak, Ram Samujh, Sandhya \\ Yaddanapudi and Abhishek Merwara
}

\section{Department of Pediatric Surgery, Postgraduate Institute of Medical Education and Research, Chandigarh}

Background and aim: Pediatric hydatid disease is rare and the commonest site involved is the liver followed by lungs. Retro-vesical pouch involvement is generally secondary with an incidence of 0.1 to $0.5 \%$ only. We report an extremely rare case of hepatic and retrovesical hydatid cyst which was managed laparoscopically.

Materials and methods: A 6-year-old male child presented to us with history of pain in the lower abdomen for 20 days. There was no history of bowel or bladder disturbances. Clinical exam was essentially normal. Contrast enhanced computed tomography (CECT) abdomen showed a $5.6 \times 5.7 \mathrm{~cm}$ well defined lesion with a cyst within cyst appearance postero-superior to the bladder and another lesion in segment 2 of liver measuring $3.8 \times 2.5 \mathrm{~cm}$ with a possibility of hydatid cyst. Hydatid serology was negative. He was started on Albendazole initially and was later taken up for surgery. A $10 \mathrm{~mm}$ infraumbilical optical port and three $5 \mathrm{~mm}$ working ports were inserted in left hypochondrium and bilateral lumbar quadrants. $5 \times 6 \mathrm{~cm}$ cyst was identified behind the bladder and another $2 \times 2 \mathrm{~cm}$ cyst was noted in the left lobe of liver bulging through the liver surface. Deroofing of both the cysts was done after aspirating cyst fluid, taking adequate precautions to avoid spillage.

Results: Feeds were established and child was discharged on postoperative day 3 on Albendazole. Child is doing well at 6 months follow up.

Conclusions: Laparoscopic deroofing is a safe and feasible technique for management of multiple hydatid cysts and provides the advantages of minimal access surgery to the patient while ensuring complete clearance with minimal spillage of cystic contents.
26.

\section{A rare case of peritoneal inclusion cyst managed} laparoscopically

\author{
Nellai Krishnan Subramonian, Anjan Kumar Dhua, \\ Prabudh Goel, Devendra Yadav, Devasenathipathy \\ Kandasamy and Minu Bajpai
}

Department of Pediatric Surgery, All India Institute of Medical Sciences, New Delhi

Background and aim: To present a case of peritoneal inclusion cyst (PIC) occurring in an adolescent female who had a previous history of open abdominal surgery, which was successfully managed laparoscopically.

Materials and methods: A 14-year-old adolescent girl presented with complaints of vague lower abdominal pain of 3 months duration. She had a history of ileal perforation 8 years prior, which was managed by laparotomy and primary closure. She had attained menarche 2 years back and had regular menstrual cycle. On examination, other than a well healed supra-umbilical transverse scar the rest of the abdominal examination was unremarkable. A transabdominal ultrasound of the pelvis revealed a large cyst in relation to the left adnexa. Magnetic resonance imaging of the abdomen showed a T2 hyperintense cystic lesion of size $6.8 \times 5.2 \times 8.7 \mathrm{~cm}$ in the left adnexa with thin septations. Uterus and both ovaries were visualised separately. Tumour markers were normal. The patient underwent diagnostic laparoscopy and laparoscopic partial excision of the cyst. Intra-operatively, a large thin walled fluid containing cyst was seen which was posteriorly and inferiorly adhered to the sacral hollow and medially to the left wall of the rectum. Left ovary and uterus could be seen separately in relation to the left lateral wall of the cyst. However, right ovary was obscured due to dense adhesions. A part of the anterior wall of the cyst was excised and sent for histopathological analysis which showed a fibro collagenous cyst wall lined by columnar epithelial cells, suggestive of PIC.

Results: No intra/post-operative complications were encountered and patient allowed orally on the same day of the surgery and discharged in stable condition. Cosmetic result was excellent.

Conclusions: PICs may occur in young female adolescents with predisposing conditions like previous abdominal surgery.

\section{7.}

\section{Laparoscopic trans uretero-ureterostomy for unilateral primary obstructive megaureter}

\section{Ashwani Mishra, Ankur Mandelia and Yousuf Siddiqui}

Department of Pediatric Surgery, Sanjay Gandhi Postgraduate Institute of Medical Sciences, Lucknow

Background and aim: We describe the feasibility and results of laparoscopic trans uretero-ureterostomy for a case of unilateral primary vesico-ureteric junction obstruction (VUJO).

Materials and methods: A male baby with an antenatal diagnosis of bilateral hydro-uretronephrosis (HDUN) presented to us with abdominal distension. Urinary stream was normal. Ultrasonography and computed tomography (CT) urography showed bilateral HDUN 
(right $>>$ left) with normal bladder wall thickness. Voiding cystourethrogram (VCUG) was normal with no reflux. LLEC scan showed findings suggestive of right vesico-ureteric junction obstruction (VUJO) with split renal function (SRF) of 24\%. Cystoscopy showed non-visualization of right orifice in the bladder. On exploration, right ureter was grossly dilated $(>5 \mathrm{~cm})$ and was opening in an ectopic location below the bladder neck with complete obstruction. A right end ureterostomy was fashioned.

Results: At 18 months of age, the baby was thriving well with good urine output, both through ureterostomy and urethra. USG showed mild right HDN with persistent dilatation of right ureter (dia.: $1.5 \mathrm{~cm}$ ). LLEC scan showed normal drainage in both kidneys with right SRF of $27 \%$. The child was planned for a laparoscopic trans uretero-ureterostomy procedure. The essential surgical steps were: (1) Recipient ureter (left) was stented. (2) Donor ureter (right) was dissected and mobilized adequately, preserving its vascularity. (3) Donor ureter was passed through a tunnel under the recto-sigmoid mesentery. (4) A longitudinal ureterostomy at the medial aspect of the recipient ureter. (5) End (right) to side (left) uretero-ureteral anastomosis was performed, avoiding tension or angulation. (6) Peritoneal windows were closed. Operating time was $210 \mathrm{~min}$ with minimal blood loss. Post-operative recovery was uneventful. Renal parameters are stable at follow up.

Conclusions: In select cases where uretero-neocystostomy of a dilated ureter is technically difficult, laparoscopic trans ureteroureterostomy presents a feasible, safe and effective option which is associated with minimal blood loss and postoperative pain, faster recovery and excellent cosmetic result.

Declaration: COI: None, Presented in a shorter format in SPUCON 2020.

28.

\section{Robotic left nephron sparing surgery}

\section{Zain Tamboli and M. S. Ansari}

\section{Sanjay Gandhi Postgraduate Institute of Medical Sciences, Lucknow}

Case presentation: Robotic left nephron sparing surgery in a 4 year old child with Wilms tumour who presented with hematuria and was evaluated to have a $(8.4 \times 6.4 \mathrm{~cm})$ left renal mass which was downsized to $(4.5 \times 3 \mathrm{~cm})$ with neoadjuvant chemotherapy with EE4A regimen,leading to an uncomplicated intra-operative and postoperative course.

Declaration: Presented at IAPS 2020.

\section{Thoracic session}

29.

\section{Bilateral diaphragmatic eventration-a diagnostic dilemma}

\section{Abhinav Sihag and Lakshmi Sundararajan}

\section{Department of Pediatric Surgery, Kanchi Kamakoti Childs Trust Hospital, Chennai}

Background and aim: Unilateral diaphragmatic eventration is diagnosed by comparing position of diaphragm versus contralateral normal dome on chest X-ray/ultrasonography/fluoroscopy. However, diagnosing and decision making for surgery in case of bilateral eventration is difficult. Thoracoscopic plication is recognized as the approach of choice in eventration. We herein highlight the pitfalls in diagnosing a case of congenital bilateral diaphragmatic eventration who improved after bilateral thoracoscopic plication.

Case presentation: A male baby born by emergency LSCS at 34/40 gestation had respiratory distress at birth, needing mechanical ventilation. Though initial x-ray showed both domes of diaphragm highly placed (Right $>$ left), later x-rays both on IPPV and HFOV showed diaphragm normally placed. Child needed prolonged ventilation and HFOV (persistent high ventilator pressures) with multiple failed attempts to extubate. Echocardiography/ultrasonography (USG)/magnetic resonance imaging (MRI) brain were normal and high resolution computed tomography (HRCT) chest was negative for lung pathology. After above, diaphragmatic weakness was considered as probable cause. USG chest for diaphragm uncontributory as child unable to tolerate spontaneous breathing. Child awaiting genetic tests for suspected myopathy. Right thoracoscopic plication was planned to see for possible improvement. Intra-operatively, entire right diaphragm was lax and thin. As ventilation improved significantly after plication, sequentially left thoracoscopic plication also done after 3 days. Child successfully extubated on postoperative day 5 and improved further progressively.

Conclusions: Bilateral congenital diaphragmatic eventration is extremely difficult to diagnose when neonate on positive pressure mechanical ventilation. Decision to operate should be carefully sought after ruling out other causes of respiratory distress.

\section{0.}

\section{Thoracoscopic thoracic duct ligation for chylothorax}

\author{
Abhishek Reddy, A. Q. Nabeel, M. M. Zameer, C. Vinay, \\ Sanjay Rao and Ashley D'Cruz
}

\section{Department of Pediatric Surgery, Narayana Health, Bangalore}

Background and aim: To describe the indications, technical aspects and outcomes of thoracoscopic ligation of thoracic duct and management of children with chylothorax.

Materials and methods: This is a prospective observational study of children who developed chylothorax. All children who presented with chylothorax from 2010 to 2021 were included in the study. All children were treated as per standard protocol of initial conservative 
management and Surgical intervention if they did not respond. Surgical intervention includes thoracoscopy with thoracoscopic thoracic duct ligation and or pleurodesis.

Results: Sixty-two children were included in the study. The cohort had a median age of 17 months (range 1 month-16 years). Median weight of $7 \mathrm{kgs}$ (range $2.4-36 \mathrm{~kg}$ ). Forty-four children responded to conservative management. Twelve patients underwent thoracoscopic thoracic duct ligation. Six had chylothorax on the right side, four had bilateral and chylomediastenal and left in one patient. Median postoperative duration of ICD in patients who underwent thoracoscopic thoracic duct ligation was 13 days (range 7-41 days). Median duration of hospital stay post thoracoscopic thoracic duct ligation was 23 days (range 11-53 days). None of the patients had thoracoscopy related complications either intraoperatively or postoperatively. One death post operatively beacuse of cardiac dysfunticon.

Conclusions: Thoracoscopic thoracic duct ligation is an effective and safe method for treating chylothorax in children.

\section{1.}

\section{Left-sided Morgagni hernia with gastric outlet obstruction-an unusual presentation of a rare anomaly}

\section{Apoorv Singh, Anjan Dhua, Devendra Yadav, Prabudh Goel and Minu Bajpai}

\section{Department of Pediatric Surgery, All India Institute of Medical} Sciences, New Delhi

Background and aim: To present our experience of an unusual presentation of a left-sided Morgagni hernia $(\mathrm{MH})$ with gastric outlet obstruction (GOO) and its laparoscopic management.

Materials and methods: A 2-year-old male child presented to us with characteristic clinical features of GOO for three months. There was associated weight loss and wasting. There was no history of any respiratory symptoms. On a skiagram, a viscus was found to be occupying the lower right thoracic cavity. A contrast-enhanced computed tomography (CECT) revealed a right-sided $\mathrm{MH}$ with the stomach and pylorus as the hernia's content. On laparoscopy, a leftsided Morgagni hernia (in contrast to CECT findings) was found with the sac going towards the right hemithorax. The contents were reduced. The patient then underwent partial sac excision and repair of the defect. The postoperative course of the patient was uneventful. Results: No intra/postoperative complications were encountered, and the child was allowed orally on the second postoperative day. After a follow-up of 3 months, the child is asymptomatic, and there is no recurrence.

Conclusions: A rare form of presentation of $\mathrm{MH}$ is reported in a child that was managed laparoscopically with an acceptable outcome.
32.

\section{Thoracoscopic lobectomy for congenital lobar emphysema in a child}

Tanmay Motiwala, Kirtikumar J. Rathod, Rupesh Sikchi, Sadik Mohammed, Taruna Yadav, Daisy Khera and Arvind Sinha

Department of Pediatric Surgery, All India Institute of Medical Sciences, Jodhpur

Background and aim: To describe the thoracoscopic approach in management of congenital lobar emphysema in a 1.5 years old child. Materials and methods: The index case is a 1.5 years old female, presented with complaints of cough, cold and difficulty in breathing on and off since 3 months of age. Patient was initially misdiagnosed as a case of pneumothorax and chest tube was inserted for the same at outside hospital. On clinical examination, there was hyper-resonant chest sound on left side. Chest $x$ ray was suggestive of over-expanded hyperluscent left upper lobe with mediastinal shift. High resolution computed tomography (HRCT) finding was suggestive of congenital left lobar emphysema with contra lateral trachea-mediastinal shift. Patient underwent thoracoscopic left upper lobectomy under single lung ventilation. Three $5 \mathrm{~mm}$ ports were inserted. 4th intercostal space left anterior axillary line port for camera and 3rd intercostals space and 6th intercostals space left midaxillary line port for instrumentation was created. Diseased lung lobe was identified. Artery, vein and bronchus supplying the left upper lobe was ligated separately and divided. Specimen was retrieved through lowermost port site and chest tube was inserted.

Results: Patient tolerated the procedure well and post operative period was uneventful.

Conclusions: Thoracoscopic lobectomy is a good alternative to traditional thoracotomy for children with congenital lobar emphysema as it results in reduced length of postoperative period, lesser amount of complications and good cosmetic and functional results.

\section{3.}

\section{Thoracoscopic repair of congenital diaphragmatic hernia: Mesh or no mesh}

\section{Vaibhav Pandey and A. N. Gangopadhyay}

Department of Pediatric Surgery, Institute of Medical Sciences, Banaras Hindu University, Varanasi

Background and aim: Congenital diaphragmatic hernia (CDH) occurs due to a failure in closing pleuroperitoneal membrane thus resulting in an incomplete diaphragm formation, which allows passage of the abdominal viscera into the thorax. Minimally invasive approach via thoracoscopy or laparoscopy is applicable for treatment of CDH since 1995. In this paper, we present our experience of thoracoscopic repair of $\mathrm{CDH}$ using mesh or without mesh.

Materials and methods: From 2015 to 2020, 20 patients with CDH were admitted to Pediatric Surgery Department of Institute of Medical Sciences, Banaras Hindu University. The median age at the time of repair was 3 months. Inclusion criteria were weight over $2 \mathrm{~kg}$ and stable hemodynamics and arterial blood gas. 3 cases were intubated 
before entering the operating room. The defect was in the left side except in three cases. In 8 cases, we used prolene mesh for reinforcing the closure.

Results: The mean time of operation was $80 \mathrm{~min}$ (40-230 min). Intraoperative mortality was zero. In hospital, mortality occurred in two cases due to septicemia in one and respiratory and cardiac failure in another. Conversion to open surgery was required in one case only. Late recurrence was observed in two cases. None of the children with mesh repair had recurrence. The mean time of follow up was 14.6 months (3-36 months).

Conclusions: It seems that appropriate case selection and liberal use of mesh as a part of repair may cause better results and decreased chance of early and late recurrence.

34.

\section{Robotic assisted thoracoscopic repair of congenital diaphragmatic eventration/hernia}

\section{Aniruthan, Kumaravel Sambandan, Shiju K. Sreepathy, Ketaki Gharpure and Aishwarya Malla}

\section{Department of Pediatric Surgery, Jawaharlal Institute} of Postgraduate Medical Education and Research, Puducherry

Background and aim: Congenital diaphragmatic eventration in children is treated by plication of eventrated/repair of diaphragm, the use of Robotics is rarely described. Potential drawbacks usually cited are the large instruments and small working space. A step by step video of the procedure is demonstrated along with review of the five operated cases.

Materials and methods: A 4-year-old boy with recurrent episodes of cough and respiratory symptoms since infancy. Radiograph revealed left diaphragmatic eventration. Given the persistent symptoms child was taken up for a robotic-assisted repair. Robotic-assisted thoracoscopic left diaphragmatic eventration repair was carried out using the da Vinci Xi system employing a three-port technique. The patient had oral feeds the same evening, intercostal drain removed next morning and discharged within $24 \mathrm{~h}$. He had good pain control on paracetamol alone and is well on follow up of 2 years. We noted no difficulty using either the $8 \mathrm{~mm}$ instruments or a lack of space.

Results: We have carried out four cases, the youngest a 5 month $5 \mathrm{~kg}$ baby, who also recovered in the same time frame.

Conclusions: Thoracoscopic repair of congenital diaphragmatic eventration has been reported since the late 1990s and surgeons have described limitation of movement of trocar by the rigid rib cage causing difficulty in needle handling and suturing as a drawback of the technique. Better magnification, excellent 3-dimensional high definition (3D-HD) vision and increased degrees of freedom of the robotic instrumentation help to overcome this limitation making the plication of diaphragm easier and more accurate.

\section{Urology session}

35.

\section{Urinary leak following laparoscopic pyeloplasty}

\section{T. Heera and Lakshmi Sundararajan}

Department of Pediatric Surgery, Kanchi Kamakoti Childs Trust Hospital, Chennai

Background and aim: Urinary leak after pyeloplasty is reported in $2-5 \%$ after pyeloplasty. We report two children with urinary leak following laparoscopic pyeloplasty by transperitoneal and retroperitoneal approach to highlight increased morbidity of intraperitoneal urinary leaks.

Case presentations: Patient 1: 3-year-old boy who underwent retroperitoneoscopic pyeloplasty with DJ stent insertion developed urinary leak from perinephric drain on postoperative day (POD)-1. $\mathrm{He}$ was otherwise asymptomatic and leak settled following $48 \mathrm{~h}$ of urethral catheter drainage. Total hospital stay was 3 days. Patient 2: 3-year-old girl with bilateral hydroureteronephrosis $\mathrm{R}>\mathrm{L}$, underwent Cystoscopy/RGP showing right pelvi-ureteric junction obstruction (PUJO) with vescio-ureteric junction obstruction (VUJO), requiring sequential surgery. On postoperative day- 2 after transperitoneal laparoscopic pyeloplasty with DJ stent insertion, child developed severe abdominal pain, distension with ileus, high grade fever and raised septic markers. X-ray abdoen showed stent folded within the right ureter, ultrasonography (USG) of abdomen showed perinephric urinoma. Cystoscopic right VUJ stenting and laparoscopic drainage of urinoma was performed. Urine and peritoneal culture grew pseudomonas which was treated with antibiotics for 3 weeks. Leak stopped and drain removed after 4 days. Total hospital stay was 12 days. She underwent reimplantation after 4 months. Follow up USG/DTPA showed good resolution of hydroureteronephrosis.

Conclusions: The systemic consequences and morbidity of urinary leak is different in retroperitoneal vs. transperitoneal laparoscopic pyeloplasty. However, even after urinary leak, managed appropriately with antibiotics and adequate drainage of the system/collection, the pelviureteric anastomosis can heal well. This difference in morbidity should be weighed in the discussion regarding choice of transperitoneal and retroperitoneal approach for laparoscopic pyeloplasty.

\section{6.}

\section{Ureteroscopic management of ureteric stone in children: dose size matter?}

\section{Gaurav Shandilya, Shitangsu Kakoti, K. Prabhu, Aneesh Srivastava and Mohd S. Ansari}

\author{
Sanjay Gandhi Postgraduate Institute of Medical Sciences, \\ Lucknow
}

Background and aim: The incidence of urolithiasis in pediatric and adolescent age group is much less as compared to the adult population. This research has been undertaken to study the management of ureteric stones in this age group with special reference to its clinical outcome. 
Materials and methods: Our study is a retrospective study, conducted over a period of 19 years in a tertiary care center. All patients up to 18 years of age, admitted and treated with the diagnosis of ureteric stones are included in this study. The patients were divided into two groups based on stone size (Group I $<10 \mathrm{~mm}$ and Group II $\geq 10 \mathrm{~mm}$ ). The eventual outcome was analyzed in terms of various factors including size, site, duration of surgery, stone free rate, use of stent, number of sittings, any intra-operative complication and, duration of hospital stay utilizing standard statistical methods.

Results: A total of 41 patients were eligible for this study out of which group I and group II contain 14 and 27 patients, respectively. The mean age of presentation was $12.6 \pm 4.2$ years and male to female ratio was 3:1. Left sided and lower ureteric stones were more common even though $97.5 \%$ stones were successfully removed with single sitting ureteroscopic lithotripsy with $100 \%$ stone free rate in $95 \%$ cases. The mean operating time was $56.7 \pm 21$ min with mean hospital stay of $4 \pm 1.5$ days.

Conclusions: Ureteroscopic management of ureteral stones in paediatric population is a feasible option with acceptable stone free rate and minimal complication regardless of any stone size.

37.

\section{A bridge between laparoscopic and open technique in treatment of pelvi ureteric junction obstruction for the beginners}

\section{B. Akshay Kalavant, Venkatesh Annigeri, Anil Halgeri and Prashant Zulpi}

\section{Department of Pediatric Surgery, SDM college of Medical Sciences, SDM Medical University, Dharwad}

Background and aim: To look for the feasibility of laparoscopy assisted pyeloplasty for beginners and its outcome.

Materials and methods: It's a retrospective non randomized analytical study of patient undergoing pyeloplasty in SDM college of medical sciences from June 2018 to February 2020. The patients were divided into two groups based on whether patients have under gone laparoscopic assisted approach (Group A) or open pyeloplasty (Group B). Laparoscopic assisted pyeloplasty was performed by transperitoneal technique using $15 \mathrm{~mm}$ working port and two $3 \mathrm{~mm}$ working ports as used in laparoscopic pyeloplasty. Kidney was approached after reflecting colon and renal pelvis was dissected out along with the pelviureteric junction. Ten-mm port was inserted in anterolateral region under vision. The PUJ is pulled out to the exterior through this wound and pyeloplasty was done. In case we needed a better exposure, the wound was increased to $1-2 \mathrm{~cm}$ more. The open pyeloplasty was carried out by standard technique through extraperitoneal approach from anterolateral incision. Following parameters were noted patient demographics, duration of surgery, no of days of hospital stay, kidney dimensions, wound complications and use of analgesia in the child. Following patients were excluded from the studies- redo cases, PCN catheters prior to surgery, ectopic kidneys. The above features were compared between the two groups.

Results: There were 12 patients in the laparoscopic assisted group and 16 patients in open groups. In group B the number of female patients were only one. Where as in group A $50 \%$ were females. Duration of surgery was more in group A with mean operating time of 154.16 min compared to group B of $103.33 \mathrm{~min}$. There were four patients in group A with age above 3 years, three of them had to be converted to open and one had to undergo surgery with a larger scar.
Excluding the four patients there was no difference in age group of the patients between two group. Anterior posterior diameter of the pelvis was also more comparable in the two groups. The hospital stay was more in the patient in the group A. one patient had recurrence of PUJO needing redo surgery. There was no major complication in open technique except wound infection who required number of hospital stay of 8 days. There is no significant difference between the two groups in analgesic usage.

Conclusions: Laparoscopic assisted pyeloplasty is a middle path especially for the young surgeons along with the reasonable small incision without much compromising in ureteropelvis anastomosis. The procedure is not suitable for age group above 3 years and helpful in infants. Excessive traction on the PUJ due to suboptimal exposure should avoided.

38.

A comparative study of pediatric open pyeloplasty, laparoscopy-assisted pyeloplasty and robot-assisted pyeloplasty

Reya Rachel Abraham, A. Q. Nabeel, M. M. Zameer,
C. Vinay, Sanjay Rao and Ashley D'Cruz.

Department of Pediatric Surgery, Narayana Health, Bangalore

Background and aim: To compare the postoperative outcomes of pyeloplasty in open, laparoscopic and robot-assisted approach in pediatric patients.

Materials and methods: This is a retrospective observational study. The study included children undergoing pyeloplasty in our Centre from 2016 to 2020. Postoperative outcomes were analysed, namely (1) postoperative pain management requiring opioid analgesics, (2) duration of surgery mins, (3) postoperative complications: urinary leak (those requiring intervention such as redo surgery and percutaneous nephrostomy) and wound infection and (4) hospital stay (days). Data were collected from medical records.

Results: Ninety-Six patients were included, of which seventy-five were boys. Age ranged from 1.5 to 168 months (mean age 84 months). Sixty-eight underwent open, sixteen underwent robotic and twelve underwent laparoscopic pyeloplasty. The surgical approach was decided by the surgeon's preference. Respective data is as follows: OP $(n=68)$ LAP $(n=12)$ RAP $(n=16)$; mean age (months) 23, 72, 96; mean operating time (mins) 178, 279, 300; opiod requirement $(\%)$ 20, 8, 37; postoperative complications requiring interventions 4, 0, 1; and mean hospital stay (days) 5, 4, 4 .

Conclusions: In our Institute, open pyeloplasty is preferred for children of a younger age group and the minimally invasive approaches are reserved for older children.

We conclude that the minimally invasive surgeries had less postoperative complications and shorter hospital stay as compared to the open approach. Within the MIS, LP had a lower requirement of opioids in comparison to robotic assisted pyeleoplasty. However, these gains are at an expense of higher operating costs, longer operating time and an added pitfall of a long learning curve for surgeons in the case of minimal invasive surgery. A large prospective multicentre study is required to clearly define the role of each of these approaches. 
39.

\section{Laparoscopic Pyeloplasty-our experience from a tertiary care centre}

\section{Aniruthan, Bikash Kumar Naredi, Bibekanand Jindal, Kumaravel Sambandhan and Aishwarya Malla}

Department of Pediatric Surgery, Jawaharlal Institute of Postgraduate Medical Education and Research, Puducherry

Background and aim: Pelviureteric junction (PUJ) Obstruction is one of the common urological problems encountered in pediatric age group. Laparoscopic pyeloplasty was first described in 1993 and has been widely used to correct the PUJ obstruction as an alternate to open pyeloplasty. Many studies have been published on laparoscopic pyeloplasty showing comparable outcomes with open pyeloplasty. Materials and methods: Retrospective review of 38 cases of laparoscopic pyeloplasty done between Jan 2015 and Dec 2020 in our institute. All the cases were done with three ports - one $5 \mathrm{~mm}$ umbilical port and $2 \times 3 \mathrm{~mm}$ or $5 \mathrm{~mm}$ working ports. Anderson Hynes dismembered pyeloplasty was done for all cases. Urinary catheter was removed on post operative day 1 and the drain was removed on postoperative day 2 .

Results: Total 38 cases were reviewed. Youngest patient operated was 2 months old. Mean age is 40 months. Mean duration of surgery is $283 \mathrm{~min}$. Mean hospital stay is 2 days. Mean follow up period is 3 years. Only one case was converted to open in view of malrotation. Appearance of scar is cosmetically superior.

Conclusions: Laparoscopic pyeloplasty offers many advantages over the open pyeloplasty like, less post operative pain, early recovery, shorter hospital stay and better cosmesis. The disadvantages include long operating time and prolonged anesthesia which can be overcome by experience as the learning curve falls. Operating time comes down drastically over time.

40.

\section{Retrograde intrarenal surgery technique and its feasibility in children: a single institutional experience}

Prabhu Karunakaran, Zain Tamboli, Punith Mathur, Abhiskek Shitanshu, Abhay Navneeth, Danish Gourav, Y. Priyank and M. S. Ansari

\section{Sanjay Gandhi Postgraduate Institute of Medical Sciences, Lucknow}

Background and aim: To share our experience of Retrograde intrarenal surgery (RIRS) and its outcome in paediatric population.

Materials and methods: Retrospectively analysed data of children who underwent RIRS for either primary or residual renal calculi from January 2017 to January 2021, were included in this study. A 7.5 Fr flexible ureteroscope with an access sheath was used in all cases while performing RIRS. All the patients had a stent left in situ at the end of procedure. The clinical data including stone burden, number of sittings, operative time, stone-free rate, and grade of postprocedural complications were analysed with appropriate statistical methods.

Results: A total of 20 patients were included in this study, of which $33 \%(6 / 20)$ had congenital renal anomalies. Seven patients $(35 \%)$ presented with primary renal calculi and underwent RIRS directly while rest of them $(65 \%)$ had residual calculi following other procedures like ESWL, URSL or PCNL before undergoing RIRS. The mean age of presentation was $8 \pm 1.2$ years, mean stone size was $12.6 \pm 2.2$, mean number of sittings was $1.2 \pm 1.4$ and mean operating time was $48.2 \pm 7.2 \mathrm{~min}$. The postprocedural complications were seen in 4 (20\%) patients in the form of Grade-2 modified Clavein-Dindo classification in 3 and Grade 3 in 1 patient. The $100 \%$ stone-free rate was seen in $80 \%$ of cases. One patient had to undergo concurrent Mini PCNL for infundibular stenosis.

Conclusions: With recent technical developments, minimally invasive procedure like RIRS is a safe and effective modality of treatment in paediatric patients for both primary and residual renal calculi.

\section{1.}

\section{Laparoscopic A-H pyeloplasty a modified technique of uretero-pelvic anastomosis.}

\section{Venkata Sathyanarayana Kotte}

Department of Pediatric Surgery, Niloufer hospital for Women and Children, Osmania Medical College, Hyderabad

Background and aim: To design an anastomotic technique, easier to execute, meticulous, more promising.

Materials and methods: The technique of suturing of uretero-pelvic anastomosis modified. No Prolene stitch to pelvis, suturing started at upper end of anastomosis used as a stay stitch. Continuous suturing from above downwards to the lower angle completed unlike the routine technique, with knot outside. The DJ stent passed with ease and the suturing continued from below upwards till it reaches the upper stay suture and tied over with knot outside again.

Results: Fifty two cases operated with this technique, from 1 months to 8 years age. Mean operating time $1 \mathrm{~h} 40 \mathrm{~min}$. All cases recovered well, discharged on 4th postoperative day.

Conclusions: avoiding prolene stitch minimizes risk of tare to an inflamed pelvis which sometimes could be infected. Anastomosis done with ease, confidence and more meticulously. Stent insertion is better facilitated in this technique.

\section{2.}

\section{Laparoscopic management of distal ureteric calculus in a child with obstructed megaureter}

\author{
Anis Akhtarkhavari, Ramesh Babu \\ and Venkatasubramaniam
}

\section{Department of Pediatric Surgery, Kanchi Kamakoti Childs Trust} Hospital, Chennai

Background and aim: Association of primary obstructive megaureter and distal ureteric calculus is rare. We herewith report laparoscopic ureterolithotomy and extravesical reimplantation in a child with this unusual combination.

Methods and results: A 6-year-old girl child presented with a history of left loin pain and hematuria; ultrasonogram and computed tomography (CT) renal confirmed presence of a distal ureteric calculus. The entire ureter was dialted upto the vesico ureteric junction. A uretero-renoscopy and lithoclast was planned. On cystoscopy a 
ureterocele like bulge with obliterated vesico ureteric junction was found and an attempt to open the ureteric orifice and to pass a guide wire failed. Ureteroscopy was abandoned and an extravesical laparoscopic approach was planned. The dilated distal ureter was identified and exposed, the ureter was opened, and stone was extracted. In view of the distal obstruction, the ureter was disconnected from the bladder and an extravesical reimplantation done. Post operatively catheter removed on day 3 and child was discharged on day 4. Post stent removal at 6 weeks, the child remained asymptomatic at 1-year follow-up. Calculus was made of calcium oxalate and metabolic evaluation did not reveal any specific problem.

Conclusions: In primary obstructive megaureter the juxta vesical portion of the ureter is narrow, adynamic and variably obstructed. With an impacted calculus at the lower ureter, the task of stone retrieval becomes impossible uretreoscopically. With expertise and astute surgical skills, a laparoscopic approach is a good alternate minimally invasive management.

\section{3.}

\section{Laparoscopic pyeloplasty for pelvi-ureteric junction obstruction of lower moiety in a partial duplex system: technical considerations}

\section{Shyamendra Pratap Sharma, Ankur Mandelia, Rohit Kapoor and K. Pujana}

\section{Department of Pediatric Surgery, Sanjay Gandhi Postgraduate Institute of Medical Sciences, Lucknow}

Background and aim: Management of pelvi-ureteric junction obstruction (PUJO) of lower moiety in a partial duplex system is technically challenging as dissection at the pelvis may jeopardize the vascularity of the normal moiety ureter. Also, the length of the lower pole ureter is the major determinant factor for the selection of standard pyeloplasty vs. pyelo-ureterostomy. We report the feasibility and efficacy of laparoscopic pyeloplasty in such a case.

Materials and methods: A 9 month old boy with an antenatally diagnosed right hydronephrosis (HDN) presented to us with a palpable renal lump. Ultrasonography showed right HDN with pelvic APD of $28 \mathrm{~mm}$. LLEC scan revealed right duplex with lower moiety PUJO with $40 \%$ split renal function (upper moiety $65 \%$, lower moiety $35 \%$ ). The patient was planned for a laparoscopic dismembered pyeloplasty.

Results: Laparoscopy was performed using three ports. The right colon was reflected medially. Dissection of the dilated pelvis revealed an incomplete duplex system. The pelvis of the lower moiety was dilated with a short non-dilated ureter joining the upper moiety ureter. The narrowed PUJ segment of the lower moiety was excised and the lower moiety ureter was spatulated over its lateral aspect into the common ureter. During this step it was important not to devascularise the upper moiety ureter. Wide, tension free, pelvi-ureteral anastomosis was fashioned with 5-0 absorbable sutures over a $3 \mathrm{~F}$ double-J stent. Operating time was $110 \mathrm{~min}$. Post-operative course was uneventful. DJ stent was removed at 6 weeks. On follow-up, the patient is asymptomatic with preserved cortical function and unobstructed drainage of right kidney.

Conclusions: Laparoscopic pyeloplasty for lower moiety PUJO in a partial duplex system enables fine tissue dissection, minimal handling of normal ureter and precision in suturing; overcoming the potential challenges involved in the minimally invasive management of such a case.

\section{Oncology and miscellaneous session}

44.

\author{
Minimal access approach to neuroblastoma. The \\ gateway to MIS in pediatric oncology!!
}

\author{
Ashutosh Kumar, Nitin James Peters, Ram Samujh, \\ R. Shubhalaxmi Nayak, Muneer A. Malik, Amita Trehan \\ and Deepak Bansal
}

Department of Pediatric Surgery, Postgraduate Institute
of Medical Education and Research, Chandigarh

Background and aim: To determine the role and outcome of minimal invasive surgeries in patients with neuroblastoma.

Materials and methods: A total of six patients were included in this report. 4 patients had abdominal neuroblastoma, of which thtee had left sided supra renal masses and one was a right suprarenal tumor. All these patients except two underwent neoadjuvant chemotherapy. All these four cases were managed laparoscopically. There were two patients who underwent thoracoscopic excision of neuroblastomas. The outcomes were assessed in terms of completion of the procedure, time taken to operate, blood loss, post-operative analgesia requirement, days to discharge, histopathology and a minimum follow up of 1 year.

Results: The mean age was 1.8 years. There were three girls and three boys recruited in this series. The mean operative time was $140 \mathrm{~min}$. All the procedures were completed by the MIS approach. Mean day to discharge was 2.6 days. The HPE revealed differentiation neuroblastoma in five and undifferentiated neuroblastoma in one patient. There was no recurrence at the end of the study.

Conclusions: MIS resection for carefully selected neuroblastoma are associated early recovery, early discharge and less blood loss, decreased number of hospital days. Optimal oncological resections can be achieved with a good short term outcome.

\section{5.}

\section{Laparoscopic right adrenalectomy for a rare feminising adrenocortical tumor}

\section{Yusuf Siddiqui, Ankur Mandelia and Ashwani Mishra}

Department of Pediatric Surgery, Sanjay Gandhi Postgraduate Institute of Medical Sciences, Lucknow

Background and aim: Experience with laparoscopic adrenalectomy for adrenal tumors in children is limited. We report the feasibility and outcome of laparoscopic right adrenalectomy for a large, feminising adrenocortical tumor in a 3 years old girl.

Materials and methods: A 3 years old girl presented to us with cyclical bleeding per vaginum of 3 months duration. Physical examination revealed breast enlargement, clitoromegaly, pubic and axillary hair development. Blood pressure was normal for age. Endocrinological work up revealed high serum estradiol (206 pmol/ L) and high serum DHEA ( $>27$ umol/L). CT abdomen showed a $6 \times 5 \times 4.5 \mathrm{~cm}$, homogenous mass in the right adrenal gland. 
Results: Laparoscopic right adrenalectomy was performed by the lateral trans-peritoneal approach using four ports. The liver was retracted medially after division of the right triangular ligament, exposing the upper pole of the kidney, the adrenal and the vena cava. Dissection was initiated on the lateral border of the vena cava in order to visualize, clip and divide the main adrenal vein. An accessory adrenal vein was also dissected, clipped and divided. Dissection of all aspects of the adrenal gland was performed, cauterizing and dividing multiple small arterial branches. After complete dissection, the adrenal gland was placed in an endo-bag and removed intact through an augmented port incision. The procedure was uneventful, lasting $140 \mathrm{~min}$. The child was allowed orally on the same day, had minimal analgesia requirements and was discharged after $72 \mathrm{~h}$. Histopathology revealed an adrenal adenoma with Weiss' score of 3. At 18 months follow up, the patient is asymptomatic with normal endocrinological and imaging parameters.

Conclusions: Laparoscopic adrenalectomy is safe and effective in children with well-circumscribed, benign adrenal masses. The lateral trans-peritoneal approach offers optimal visualization and excellent outcome in terms of minimal discomfort, rapid recovery, and excellent cosmesis.

\section{6.}

\section{Robotic surgery in bilateral angiomyolipoma}

\section{T. Vidhya and V. Sripathi}

\section{Apollo Children's hospital, Chennai}

Background and aim: To present the role of robotic surgery in bilateral renal angiomyolipoma.

Materials and methods: A 13 years obese boy with tuberous sclerosis was incidentally detected with bilateral upper pole mass in the kidneys in ultrasound on regular follow. He was asymptomatic, normotensive with normal renal function, his contrast enhanced computer tomography (CECT) revealed bilateral upper polar mass $(\mathrm{R}>\mathrm{L})$ with fat stranding? Renal cell carcinoma, however fat seen in the left side was highly favourable for an angiomyolipoma. Oncologist recommended tissue diagnosis but Radiologist and Interventional radiologist deferred biopsy in view of the possibility of massive haemorrhage. Robotic enucleation of smaller lesion on the left side was planned and carried out with a console time of $180 \mathrm{~min}$ and blood loss of $250 \mathrm{ml}$. After occlusion of renal artery, with the help of intraop ultrasound and nephrometry scoring, border of lesion was marked $(3 * 3 \mathrm{~cm}$, highly vascular, feeders from aorta), cored out and excised. Post operative period was uneventful, discharged on postoperative day 4.

Results: HPE revealed Angiomyolipoma. There was excellent renal recovery on the left side indicating no recurrence, the larger lesion on the right side was stable during 1 year follow up and planned for angioembolisation.

Conclusions: Robotic surgery is an elegant solution in complicated scenarios, as in this case, it provided enough tissue for histopathology and also helped to remove the lesion with minimal disturbance to the child.
47.

\section{Laparoscopic cortical preserving adrenalectomy for right sided pheochromocytoma}

\author{
Biangchwadaka Suchiang, Rahul Saxena, Arvind Sinha, \\ Kirtikumar Rathod,Taruna Yadhav and Madhukar Mittal
}

Department of Pediatric Surgery, All India Institute of Medical Sciences, Jodhpur

Background and aim: Pheochromocytoma is a rare disorder in the pediatric age group seen between 11 and 13 years of age. It has a slight predilection to the male population and one of the rare causes of correctable hypertension in children. Diagnosis is made by a combination of radiological imaging and clinical features. Management consists of a surgical excision. Here we present a case of pheochromocytoma in an adolescent in whom laparoscopic tumor excision was done.

Case presentation: A 15-year-old boy was referred to our institution with complains of headaches, palpitations and occasional abdominal pain. He was found to have severe hypertension, and radiological imaging and MIBG suggested an avid right adrenal mass i.e. pheochromocytoma; thereby explaining his symptoms. He was planned for tumor excision after confirming the absence of any metastasis and was started on alpha and beta blockers as a part of the pre-operative preparation. After adequate control of blood pressure and heart rate, he was taken for laparoscopic excision of the tumor. Via four ports, a very careful dissection using various energy sources was done as the tumor was in close proximity to the IVC. Tumor was removed with an intact capsule leaving behind normal right adrenal tissues.

A brief period of hypotension was present intra-operatively after tumor excision from which the child recovered shortly and was eventually discharged on the 5th post-operative day. One month later an MIBG scan was done which did not suggest any area of active malignancy, along with a drop-in level of normetanephrines. The blood pressure however is still on the higher side which is otherwise under control with prazosin and metoprolol.

Conclusions: Laparoscopic excision of pheochromocytoma is feasible in the pediatric age group and should be considered over the open procedure. Along with having the advantages of minimal invasive surgery (MIS) as a whole, it is also able to adhere to tumor excision protocol where a complete tumor excision was achieved along with sparing the normal adrenal tissue.

48.

\section{Endoscopic third ventriculostomy with or without choroid plexus ablation: technique and results}

Vaibhav Pandey and A. N. Gangopadhyay

Department of Pediatric Surgery, Institute of Medical Sciences, Banaras Hindu University, Varanasi

Background and aim: Pediatric hydrocephalus is a significant contributor to infant morbidity and mortality, particularly in developing countries. The mainstay of treatment has long been shunt placement 
for CSF diversion, but recent years we have seen the rise of alternative procedures such as endoscopic third ventriculostomy (ETV), which provides similar efficacy in selected patients. The addition of choroid plexus ablation (CPA) to ETV has been proposed to increase efficacy, but the evidence of its utility is limited. This study is aimed to determine the efficacy and safety of ETV + CPA in comparison to ETV alone for the treatment of hydrocephalus.

Materials and methods: From 2015 to 2020, 25 patients with hydrocephalus were admitted to Pediatric Surgery Department of Institute of Medical Sciences, Banaras Hindu University. The median age at the time of operation was 1 year. Inclusion criteria were all patients with obstructive non communiating hydrocephalus. 14 cases were done ETV alone and in 11 cases CPA + ETV was done. The primary outcome was success rate, defined as no secondary procedure required for CSF diversion; secondary outcomes included time to failure, mortality, and complications.

Results: Overall, there was no significant difference in success rates between ETV and ETV + CPA (RR 1.24, 95\% CI 0.88-1.75, $\mathrm{p}=0.21$ ).

Conclusions: The immediate post-operative CSF leak was less in the ablation group.

\section{9.}

\section{Thoracoscopic excision of ganglioneuroma in ROHHAD syndrome}

\section{K. Shreyas, Kirtikumar Rathod, Taruna Yadav, Darshana Rathod, Shilpa Goyal and Arvind Sinha}

\section{Department of Pediatric Surgery, All India Institute of Medical Sciences, Jodhpur}

Background and aim: Rapid-Onset Obesity with Hypoventilation, Hypothalamic dysfunction, and Autonomic Dysregulation Syndrome (ROHHAD) is rare condition seen in pediatric population. The occurrence of the hypoventilation leading to respiratory arrest and association with neural crest tumors makes the syndrome more fatal. The role of minimally invasive surgery in the management of pediatric thoracic tumors is gaining popularity. Here we report a case of Thoracic ganglioneuroma associated with ROHHAD syndrome successfully managed by thoracoscopic excision.

Case presentation: A 4-year-old girl was presented to the hospital OPD with complaints of rapid-onset weight gain, hyperphagia, inappropriate behavior and laughter. On evaluation, the patient had persistent high blood pressure $(95+12$ centile) for which antihypertensive was given. The child was evaluated for the cause of obesity in which the biochemical parameters were found to be normal. To rule out the central cause of obesity, MRI brain was done which showed features of paraneoplastic cerebellitis. The involvement of the cerebellum and midbrain prompted screening of spine, which revealed a well- defined left paravertebral lesion which was consistent with left paravertebral ganglioneuroma and thus, the diagnosis of ROHHAD syndrome was established. Patient successfully underwent thoracoscopic excision of the thoracic mass despite of its close proximity to the arch of aorta and the subclavian vessels. The histopathology findings were consistent with ganglioneuroma. The patient is on regular follow-up for past 6 months with drastic improvement in behavioral pattern and routine activities and persistent hypertension.

Conclusions: We conclude that thoracoscopy is a feasible and relatively safe procedure thoracic tumors like ganglioneuromas even in a complex and challenging conditions like ROHHAD syndrome. It imbibes added advantages over open procedure like a short hospital stay, decreased risks of post-operative infections, pain and also cosmetically acceptable incisions.

\section{0.}

\section{Endoscopic management of vocal cord palsy in children}

Avantika Tiwari, A. Q. Nabeel, M. M. Zameer, C. Vinay, Sanjay Rao and Ashley D'Cruz.

\section{Department of Pediatric Surgery, Narayana Healthcare, Bangalore}

Background and aim: To study the outcome in children with vocal cord palsy.

Material and Methods: This is a retrospective observational study. Data was collected from medical records of children who were diagnosed at our centre to have vocal cord palsy from 2012 to 2021. Diagnosis was made on MLB and tracheostomy was done. In children with no resolution of vocal cord palsy, endoscopic vocal cord lateralisation was performed.

Results: A total of 14 children were diagnosed to have vocal cord palsy, on MLB. Age ranging from 2 days to 15 years. Five of them were girls. All children had presented with an initial complaint of stridor. Three of them presented with respiratory distress and weak cry. On evaluation nine children were found to have idiopathic palsy, three developed palsy post mediastinal surgery (two cardiac), two had Arnold chiari malformation. Three children had unilateral vocal cord palsy. Thirteen children underwent tracheostomy. Twelve children were on oral feeds and had no aspiration. One child had severe gastroesophageal reflux (GER) and underwent gastrostomy + fundoplication. Children were on regular follow up. Five children required vocal cord lateralisation while eight have recovered spontaneously. One child is still on tracheostomy, awaiting follow up. Twelve children have been decannulated. Average age of children who were managed conservatively was 66.5 days. Average age of children who underwent vocal cord lateralisation is 8.2 years. The voice remains husky in all children post lateralisation.

Conclusions: Vocal cord palsy is an uncommon cause of stridor in children. A MLB is required to diagnose this condition. Majority of the cases are idiopathic. Generally, children who present in infancy recover spontaneously. While a few select cases who present later require endoscopic vocal cord lateralisation. Tracheostomy is required to secure the airway and to provide time for the palsy to recover. On the whole, outcomes are good and most will be decannulated. Voice however remains abnormal. 
51.

\section{Rigid esophagoscopy with topical Mitomycin C application for treatment of benign esophageal stricture in children: our experience}

\author{
Raghav Narang, Simmi K. Ratan, Shasanka Shekhar \\ Panda, Sujoy Neogi, Satish K. Agarwal Siddharth \\ Srivastava and Sonia Wadhawan
}

\section{Department of Pediatric Surgery, Maulana Azad Medical College, New Delhi}

Background and aim: The use of Mitomycin C local application in esophageal stricture following esophagoscopy has yielded equivocal results especially for longer stricture. We herein share our experience for the same.

Materials and methods: Five children aged 3-10 years with esophageal stricture due to various causes over past 4 years period were included, if the stricture did not yield to two consecutive endoscopic balloon dilatation or was labelled as non negotiable and patient remained symptomatic. For each patient mitomycin $(1 \mathrm{mg} / \mathrm{ml})$ was locally applied at the beginning of the esophageal stricture with a wet pledget held with biopsy forceps passed through rigid endoscope. The number of dilatation was dictated by the achieved response gauged as amelioration of symptoms and radiological improvement (for not more than three dilatations).

Results: The median age of subject was 6 years with median follow up of 28 months. Causes of esophageal stricture were post corrosive poisoning (2), post tracheo-esophageal fistulea (TEF) repair (1), post foreign body ingestion (1) and post disc battery ingestion (1). The mean time taken for procedure was $15 \pm 5 \mathrm{~min}$ and no post operative complications were noted. Mean number of Mitomycin $\mathrm{C}$ application required were $2 \pm 0.7$. Four out of five patients had complete resolution of their symptoms. One patient with stricture due to battery ingestion had to undergo Gastric pull up surgery.

Conclusions: Though endoscopic balloon dilation is the gold standard for benign esophageal stricture management, rigid esophagoscopy and mitomycin local application in selected cases can save the need for complex surgeries.

52.

\section{Endoscopic third ventriculostomy for management of non-communicating hydrocephalus in pediatric age group}

\section{Punit Singh Parihar and Ramesh Tanger}

\section{Department of Pediatric Surgery, SMS Medical College, Jaipur}

Background and aim: Non-communicating hydrocephalus (aqueductal stenosis) is a common condition in paediatric age group, mostly managed by ventriculoperitoneal shunt. But this procedure has its own inevitable complication which compel for second surgery. Endoscopic third ventriculostomy is an alternative especially for noncommunicating hydrocephalus with good clinical outcome.

Materials and methods: Patients with radiological diagnosis of noncommunicating hydrocephalus (aqueductal stenosis), MRI/CT-Brain, with no significant distortion of ventricular anatomy are the candidate for endoscopic third ventriculostomy.

After assessing the cerebrospinal fluid (clear, no turbidity, no haemorrhage), Neuroendoscope is inserted in lateral ventricle, foramen of monro identified then entered into third ventricle. The base of third ventricle is fenestrated which permits the third ventricle to communicate with the basal cistern, allowing the CSF to bypass obstruction distal to the third ventricle.

Results: Endoscopic third ventriculostomy is one of the better surgical intervention for management of non- communicating hydrocephalus in pediatric age group. Previous study showed the success rate of $80-90 \%$.

Conclusions: The advantage of ETV are restoration of physiological cerebrospinal fluid (CSF) circulation, the absence of foreign material, lower incidence of late complications It can also be used as a better alternative for previous shunt surgery with shunt malfunction. The only pre- requisite is that the ventricular anatomy should not be distorted.

\section{PESI-IAPS video award session}

\section{3.}

\section{Laparoscopic splenectomy for giant pseudocyst of the spleen}

\author{
K. Pujana, Ankur Mandelia, Rohit Kapoor \\ and Shyamendra Sharma
}

Department of Pediatric Surgery, Sanjay Gandhi Postgraduate Institute of Medical Sciences, Lucknow

Background and aim: Splenic cysts in children are rare. We report an interesting case of giant splenic pseudocyst (SP) managed by laparoscopic splenectomy (LS).

Materials and methods: A 9 years old girl presented with history of vague abdominal pain and upper abdominal fullness for past 2 years. She could not recall any abdominal trauma in the past. Physical examination revealed non-tender splenomegaly reaching up to $4 \mathrm{~cm}$ from the left costal margin. CT abdomen showed a large cystic lesion in the spleen of size $17 \times 16 \times 12 \mathrm{~cm}$. Most of the spleen was replaced by the cystic lesion, except for a small part of the lower pole. ELISA for hydatid serology was negative. LS was planned in view of the huge size of the cyst with minimal residual splenic tissue.

Results: LS was performed by lateral approach using four ports. First, the huge cyst was decompressed and $3 \mathrm{~L}$ of brownish fluid was aspirated. The dissection started by dividing the spleno-colic ligament, followed by the gastro-splenic ligament. The splenic artery and vein were dissected at the hilum, clipped and divided, taking care not to injure the tail of the pancreas. Finally, the spleen was dissected from the diaphragm and left lobe of liver to which it was densely adhered. The specimen was placed in an endobag and removed by morcellation through the $10 \mathrm{~mm}$ port. The procedure was uneventful with an operating time of $190 \mathrm{~min}$. Postoperatively, the patient was allowed liquid diet on the same day, the recovery was uneventful and she was discharged on day 3 . The patient is asymptomatic at 6 months follow up. Histopathology showed fibro-collagenous tissue lining the cyst wall with no definite epithelial lining suggestive of SP.

Conclusions: LS for giant SP is feasible and safe and offers all the advantages of minimal access surgery. 
54.

\section{Isolated extra calyceal renal hydatid cyst: management} by laparoscopic Port-in-cyst technique

\section{Department of Pediatric Surgery, Amit Pandey and Ravi Prakash Kanojia}

\section{Postgraduate Institute of Medical Education and Research, Chandigarh}

Background and aim: Isolated extra calyceal Renal hydatid cysts are rare and comprise only $2-3 \%$ of all visceral hydatid infestation. Usually, the cyst is solitary and involves the cortex of the kidney. It presents as flank pain and palpable lump. Rarely patients may also have hematuria, hypertension and hydatiduria. Surgical management is the treatment of choice and involves cystectomy, deroofing and endocyst removal and nephrectomy in cases of large cysts. There are few case reports and case series available in literature which demonstrate the feasibility of minimally invasive techniques for the surgical management of renal hydatid cyst. We present our video of a case of Left renal hydatid cyst managed laparoscopically by port in cyst technique.

Materials and methods: The index patient is a 10 years old boy presented with pain and lump in left flank since 2 months. Clinically had a large $10 \times 6 \mathrm{~cm}$ palpable lump in left hypochondrium and lumbar region. Ultrasonography and contrast enhanced computer tomography (CECT) abdomen showed $11 \times 8 \mathrm{~cm}$ large cyst in left kidney suggestive of hydatid cyst. Patient underwent laparoscopic aspiration with cyst deroofing and evacuation of hydatid cyst. A previously published Port in cyst technique was used to achieve a spill free excision of membranes. The presented video demonstrates the same.

Results: The procedure was completed without spillage and complete excision of membrane's were achieved. There was no communication with the calyceal system. The cyst dome was partly excised to deroof the cyst. Capitonnage was done by filling in the omentum. A drain was also placed in the cavity which was removed on day 3 . The follow up duration of this patient is $>2$ years without any recurrence. Conclusions: Isolated extra calyceal renal hydatid cysts can be managed like any other visceral hydatid infestation. Port-in-cyst technique provides an excellent way to achieve a spill free excision of membranes. The video presents a complete demonstration of the procedure with technical details.

\section{5.}

\section{Superior mesenteric artery syndrome laparoscopic duodeno-jejunostomy}

\section{Venkata Sathyanarayana Kotte}

\section{Department of Pediatric Surgery, Niloufer hospital for Women} and Children, Osmania Medical College, Hyderabad

Background and aim: To share our experience of management of superior mesenteric artery (SMA) syndrome by minimal access approach which is a rare condition in children, where many of our pediatric surgeons have not come across.

Materials and methods: Seven years old boy presented with intermittent pain abdomen, non-bilious vomiting, loss of weight. On evaluation with angio-computed tomography, diagnosis confirmed showing thirrd part of duodenum forked and compressed between abdominal aorta and superior mesenteric artery- SMA syndrome. Laparoscopic duodeno-jejunostomy performed with the help of three $5 \mathrm{~mm}$ ports in $3 \mathrm{~h}$ time. Four-layer anastomosis done with 3-0 mersilk suture, all hand sewn. First and 4th layer continuous suture and second and 3rd layer interrupted suturing. No drain kept.

Results: Postoperative period uneventful. Feeds started 5th postoperative day (POD), discharged 8th POD. Patient on follow up asymptomatic, with good weight gain.

Conclusions: SMAS is a rare condition in children which we could manage by MAS approach successfully, and can advocated as a procedure of choice, owing to it's advantage.

\section{6.}

\section{A complex bilobed choledochal cyst excision: a technical challenge}

\section{Ayushi Vig, Kirtikumar J. Rathod, Avinash Jadhav, Taruna Yadav, Shilpa Goyal, Vaishnavi and Arvind Sinha}

Department of Pediatric Surgery, All India Institute of Medical Sciences, Jodhpur

Background and aim: Hepatobiliary surgeries in children can be extremely challenging due to numerous anatomical variations. Here we present an extremely complicated bilobed Type 4 Choledochal cyst in a child which was managed laparoscopically.

Case presentation: A 2-year-old female presented with complaints of lump abdomen for seven months and a recent onset jaundice and fever. MRCP was suggestive of gross dilation of entire biliary tree (both intra and extra hepatic part) with symmetrical fusiform dilation of common bile duct measuring $4 \mathrm{~cm}$ in diameter, at its retro-pancreatic segment. Another large, para duodenal cystic lesion measuring $26 \times 32 \times 27 \mathrm{~mm}$, closely abutting second part of duodenum, inferior to cystic duct, was reported as a duodenal duplication cyst. A $12 \mathrm{~mm}$ camera port and three $5 \mathrm{~mm}$ working ports were inserted at infraumbilical, right lumbar, right iliac fossa and left hypochondrium respectively. Choledochal cyst visualized and dissected circumferentially. Distal end of cyst was badly adherent to the neck of pancreas which was separated using harmonic and cyst was excised. However intraoperatively it was noted that para duodenal cystic lesion reported in MRI was another lobe of the same choledochal cyst and was densely adhered to the liver. It was separated, however a part of accessory (intrahepatic) posterior wall left and burned with cautery. Hepatico-duodenostomy performed. The patient had a smooth postoperative transit. Duration of surgery was slightly prolonged due to complex anatomy and dense adhesions; however the patient's family was pleased with the excellent cosmesis and accelerated recovery. Conclusions: Choledochal cyst has multiple anatomical variations. The Para duodenal cyst which was reported as duodenal duplication cyst on radiology was intraoperatively noted to be a second lobe of the choledochal cyst itself. Careful fine dissection is the path to be followed in such difficult cases. We believe that complex hepatobiliary procedures are suited for laparoscopic surgery in children owing to the magnified view and precise dissection. However each case can be a new challenge for the surgeon. 
57.

\section{Laparoscopic excision and hepaticojejunostomy for a choledochal cyst with acute presentation}

\author{
Satish Kumar Kolar Venkatesh, Ashok Rijhwani, \\ N. Anil Kumar, Naveen Thomas, Asha Thomas, \\ Rahul Krishnan and Arpit Kumar
}

Bangalore Baptist Hospital, Bangalore

Background and aim: The ideal treatment of choledochal cyst in children is elective cyst excision with hepaticojejunostomy done by open or laparoscopic technique. Early excision during acute phase may be indicated to prevent suppuration or impending perforation of the cyst. This video demonstrates a laparoscopic excision and hepaticojejunostomy done during acute presentation of choledochal cyst in a girl.

Materials and methods: A 3 years 8 months old child with 4 days of abdominal pain and jaundice had type 4 A choledochal cyst with cholangitis and pancreatitis on investigations. She was managed with antibiotics and analgesics, but pain was unrelenting. On 4th day of admission she was posted for laparoscopy under general anaesthesia. A $10 \mathrm{~mm}$ umbilical port for camera and three additional ports of $3 \mathrm{~mm}$ for instrumentation were used. The principles of open surgery were followed and cyst excised with jejunojejunostomy performed extracorporeally through umbilical port and the hepaticojejunostomy performed intracorporeally.

Results: The child recovered well postoperatively in intensive care. The drain fluid was $100 \mathrm{ml}$ over 2 days but the drain inadvertently fell off on day 3 with no consequence. The child was fed on day 3 and was on full feeds on day 4 . The child was discharged on day 7 with well-healed port sites and is for a review after 2 weeks.

Conclusions: Choledochal cysts with cholangitis are managed with antibiotics and usually undergo elective surgery on a later date. Emergency surgery may be needed if the cholangitis is not responding and this may be safely accomplished laparoscopically during acute phase.

\section{8.}

\section{Laparoscopic correction of malrotation: learning experience for beginners}

\section{Md. Fahim Ahmad and Ravi P. Kanojia}

\section{Department of Pediatric Surgery, Postgraduate Institute of Medical Education and Research, Chandigarh}

Background and aim: Malrotation is a rotational anomaly of gut with various components. The spectrum of presentation is also varied. Laparoscopic correction is a challenge for beginners with difficulties in understanding the laparoscopic anatomy. We present a video demonstration of laparoscopic correction of malrotation explaining the anatomy pre-emptively and intraoperatively. Various other technical details are also discussed.

Materials and methods: A 5 years old girl child who had presented with recurrent bilious vomiting and failure to thrive. She had on off abdominal distension also. Upper GI contrast study was done and showed dilated stomach, DJ on right side of spine, the entire small bowel was located on right side of abdomen and colon lying above the duodenum. She underwent laparoscopic correction. Three ports $10+5+5$ were used. DJ flexure was mobilized from right side and freed from Ladd's bands.

Results: Patient underwent laparoscopic correction of malrotation uneventfully. She recovered well and discharged on post-operative day-4 and now doing well without any further complication/ admissions.

Conclusions: Laparoscopic anatomy of malrotation should be clear to the operating surgeon specially the beginners. The video provides a clear understanding for handling various components of malrotation correction to achieve a successful outcome.

59.

\section{Robotic-assisted laparoscopic extravesical ureteric reimplantation in children with unilateral and bilateral refluxing megaureter-video narrative technique}

\section{Aishwarya Malla, Apurva Arora and S. Kumaravel}

Department of Pediatric Surgery, Jawaharlal Institute of Postgraduate Medical Education and Research, Puducherry

Background and aim: To video demonstrate the technical aspects of Robotic-assisted laparoscopic extravesical ureteric reimplantation. Materials and methods: Review of technical aspect of robotic-assisted laparoscopic extravesical ureteric reimplantation fine-tuned by a single surgeon, over a dozen cases using the Da Vinci Xi System. Results: Over the past several months about 12 children (18 units) underwent robotic-assisted laparoscopic extravesical ureteric reimplantation. We followed standard technique following the Lich Gregoir principle, transperitoneal using three $8 \mathrm{~mm}$ robotic ports and one $3 \mathrm{~mm}$ assistant port. Standard steps included identification of ureter at the pelvic brim, creation of a peritoneal window, gentle hooking up of the ureter and holding up with umbilical tape, railroading of the umbilical tape to the new window above the vas or the fallopian tube to safeguarding them. Dissection of the ureter until VUJ. Nerve preservation by avoiding dorsomedial dissection. Measurement of the length of the tunnel satisfying Paquin's rule. Detrusorotomy and placement of ureter in tunnel and closure with occasional bite including the ureteral wall. Bladder mucosal breach if any was closed (only 1 in 18 units). No drains were placed. Only in bilateral cases, we placed a DJ stent on a better functioning kidney before the start of the procedure in a retrograde fashion. The youngest child operated is 24 months (weight $-11.2 \mathrm{~kg}$, height $-83 \mathrm{~cm}$ ). Six underwent a unilateral procedure and the rest underwent the bilateral procedure (total 18 units). The median operating time is $173.5 \mathrm{~min}$ (unilateral-142.5 min). One child underwent additional unilateral hernia repair and one underwent excision of the Left remnant ureter. One child had a post-operative UTI with a DJ stent in situ. Mean hospital stay and catheter days being 2 days and 1.5 days respectively. No child developed urinary retention in our limited series. Conclusions: Robotic-assisted minimally invasive surgery is safe and has minimal perioperative morbidity. Steps and Technical details of Robotic-assisted laparoscopic extravesical ureteric reimplantation are demonstrated in the video in detail. 
60 .

\section{Laparoscopic ureteric reimplantation of a single-system ectopic ureter in a girl}

\section{Mohammad Imran and Vaibhav Pandey}

Department of Pediatric Surgery, Institute of Medical Sciences, Banaras Hindu University, Varanasi

Background and aim: About $80 \%$ of ectopic ureters arise from the upper pole of a completely duplicated system. Ectopic ureters draining single systems are not common, occurring only in $20 \%$ of cases. Again, ectopic ureter draining single system in case of females is extremely rare. We report a case of a 7-year-old girl having singlesystem ectopic ureter undergoing laparoscopic ureteric reimplantation.

Methods and methods: A 7-year-old girl presented with continuous dribbling of urine along with normal voiding pattern since childhood. There was no urgency, frequency, dysuria or flank pain. There was no history suggesting stress incontinence. Physical examination was unremarkable except for leakage of urine per vaginum. Urinalysis showed $2-3$ pus cells/hpf, and urine culture revealed no growth. Blood biochemical parameters were within normal limits. Ultrasonography of the kidneys, ureters and bladder (KUB) region revealed small right kidney, measuring $56 \mathrm{~mm}$, with normal cortical echotexture and corticomedullary differentiation. Left kidney was normal, and none of the ureters was dilated. Intravenous urography showed excretion of contrast through both kidneys, with delay from the right kidney. Both pelvicalyceal systems and ureters were visible and showed bilateral single system.

Results: On cystourethroscopy, urethra, bladder, left ureteric orifice were found to be normal, but right ureteric orifice was seen below the bladder neck. Patient underwent laparoscopic transperitoneal extravesical ureteric reimplantation. At 3 months' follow-up, intravenous urography (IVU) and micturating cystourethrogram (MCU) showed no obstruction and reflux.

Conclusions: Laparoscopic procedures offer reduced morbidity due to less postoperative pain, better cosmesis, earlier return of bowel function, earlier discharge.

Declaration: A similar technique presented in IAPSCON 2020.

\section{1.}

\section{Multiloculated complex mesenteric cyst-total laparoscopic resection with laparoscopic intra- corporeal end to end small bowel anastomosis in a child}

\author{
Rupesh Suresh Sikchi, Kirtikumar J. Rathod, \\ T. K. Jayakumar, Taruna Yadav, Poonam Elhance \\ and Arvind Sinha
}

\section{Department of Pediatric Surgery, All India Institute of Medical Sciences, Jodhpur}

Case presentation: To describe the method of laparoscopic excision of complex mesenteric cyst in a child. Mesenteric cysts are rare abdominal lesions with incidence of 1 in 100,000-250,000. The index case is a 6 years old male, presented with complaints of dull aching pain abdomen and non bilious vomiting on and off since past
6 months. On examination no palpable lump was felt over abdomen. USG- thin walled cystic mass with few internal septations seen anteromedial to right kidney suggestive of mesenteric cyst. CT scan$5.3 \times 6.2 \mathrm{~cm}$ cystic multiloculated infiltrating lesion along right sub hepatic region suggestive of mesenteric lymphangioma. Patient underwent laparoscopic excision of mesenteric lymphangioma with end to end jejunal anastomosis. Patient tolerated the procedure well. Patient was allowed orally on POD 3 after removal of nasogastric tube and was discharged on POD 4. Patient currently has no complaints and is alright on regular follow up.

Three $5 \mathrm{~mm}$ ports were inserted. Infraumbilical port for camera and Right iliac fossa and left epigastric port for instrumentation was created. Mesenteric cyst was identified lying in the pelvis which was located about $25 \mathrm{~cm}$ distance from DJ flexure. Cyst was excised along with $10 \mathrm{~cm}$ of jejunum and laparoscopic intracorporeal end to end single layer anastomosis was done. Specimen was placed in endobag and was retrieved through infraumbilical port site incision.

Conclusions: Laparoscopic excision of mesenteric cyst should be the preferred way for management of large mesenteric cyst in children, as it has advantage of less post operative pain, early recovery, short hospital stay and cosmetically better wound.

\section{2.}

\section{Transperitoneal and retroperitoneoscopic approach for dysfunctional moiety nephrourterectomy in renal duplication}

\section{Saswati Behera and Ravi Prakash Kanojia}

\section{Department of Pediatric Surgery, Postgraduate Institute} of Medical Education and Research, Chandigarh

Background and aim: Renal duplications are common occurrences in pediatric age group and often the dysplastic moiety is nonfunctional. The dysplastic moiety is a cause of significant morbidity from recurrent infections, pain and occasionally hypertension. In such circumstances it warrants removal. We are presenting a video demonstration how dysfunctional moiety nephro-ureterectomy can be done by both transperitoneal and retroperitoneal approaches. Various pros and cons of the two approaches are discussed.

Materials and methods: The video presents and demonstrate the two approaches in two different patients. First patient was a two-year-old child with antenatally diagnosed left hydronephrosis. Postnatally, although asymptomatic, he was diagnosed with duplex left kidney with negligible cortical function of the upper moiety and vesicoureteric reflux. He underwent excision of upper dysfunctional moiety nephroureterectomy by retroperitoneoscopic approach. Similarly, second patient is a 8 years old boy with similar presentation and was operated by transperitoneal approach. Technical details for both the patients are described in the video.

Results: The operative time were $135 \mathrm{~min}$ for retroperitoneoscopic and $210 \mathrm{~min}$ for transperitoneal approaches respectively. Blood loss was minimal. The postoperative courses were uneventful for both the children with a duration of hospitalization of 2 days and 4 days (respectively for Patient 1 and Patient 2). 3 months follow up renal scintigraphy showed no loss of cortical function or pyelonephritic changes in the remaining parenchyma.

Conclusions: Laparoscopic excision of upper dysfunctional moiety have been proved to be safe from its open counterpart. Retroperitoneoscopic approach has an added benefit of better exposure and direct access to retroperitoneal structures with minimal bowel handling, 
whereas although easier and faster, transperitoneal approach has an inherent predisposition of adhesions formation bowel trauma and longer ileus post operatively. We have larger cohort of patients to demonstrate the differences, while the index cases are the representation of the technical maneuvers in both the approaches. 